\title{
Biological Activities of Metal Complexes with Rutin and Bio-Conjugate of Citrus Extract
}

\author{
Om Prakash ${ }^{1, *}$, Babita Belal' ${ }^{2}, J_{y o t s n a}$ Dhanik $^{2}$, Anil Verma ${ }^{2}$, Hem C Joshi ${ }^{3}$, Vivekanand ${ }^{2}$ \\ ${ }^{1}$ Department of Applied Chemistry Sardar Bhagwan Singh Post Graduate Institute of Biomedical Sciences and Research, India \\ ${ }^{2}$ Department of Chemistry, College of Basic Sciences and Humanities, G.B. Pant University of Agriculture and Technology, India \\ ${ }^{3}$ Department of Plant Physiology, College of Basic Sciences and Humanities, \\ G.B. Pant University of Agriculture and Technology, India
}

Received March 19, 2020; Revised June 12, 2020; Accepted June 20, 2020

Copyright $\bigcirc 2020$ by authors, all rights reserved. Authors agree that this article remains permanently open access under the terms of the Creative Commons Attribution License 4.0 International License

\begin{abstract}
The main aim of this study is to determine the changes occurred in the antioxidant capacity and UV-protection capacity of rutin with a different redox-potential metal cation. The complexes were characterized by UV-Visible and IR spectroscopy. Rutin belongs to the flavonols and it is a glycoside form of quercetin. Chelate formation is a result of interaction of rutin with metal ions. The chelation of metals can be crucial in the prevention of radical generation, which damages target biomolecules. The interactions between flavonoids and transition metal ions to form complexes that prevent the participation of metal ions in free radical generating processes, thus exhibit an anti-oxidant behavior, metal-flavonoid complexes various biological and pharmacological activities. The coordination of Rutin (Rut) with Rut-Zr, Rut-Mo, Rut-Zn, Rut-Cu, Rut-Ni, Rut-Fe, Cit-Zn, Cit-Mo, Cit-Zr, Cit-Cu, Cit-Fe, and Cit-Ni may simulate the catalyzed centre of the hyperoxide dismutase in the metal coordinate structure, thus displayed the DPPH, hydroxyl radical scavenging activity, metal chelating, and nitric oxide (NO) and superoxide dismutase (SOD) radical scavenging. Therefore, the metal flavonoid complexes are very helpful in discovery and development of new drugs.
\end{abstract}

Keywords Rutin, Metal Chelation, Flavonoid and Metabolites

\section{Introduction}

Flavonoids are a class of secondary plant metabolites with significant antioxidant, chelating, and antimicrobial properties; hence, they have many beneficial effects on foods and health. Rutin belongs to the flavonols and is a glycoside form of quercetin. Rutin is naturally found in fruits and plants such as potatoes, onions, ginger, tomatoes, vegetables, and herbal drinks like tea (Om et al., 2018). It has peroxyl, hydroxyl, and superoxide radical scavenging effects (Sharma et al., 2013). Its health promoting effects are also well acknowledged (Pan et al., 2014; Wang et al., 2015). Daily value of rutin is around $1.5-70 \mathrm{mg} / \mathrm{kg}$, which varies as per nutritional habits and countries regulations (Kreft et al., 1999; Nakamura et al., 2000), very low aqueous solubility is the main drawbacks of rutin. Furthermore, it is not absorbed by intestinal membrane due to glycosylated structure with a disaccharide which makes it polar and large (Sharma et al., 2013). In addition to improving chemical stability, odour and taste masking, colloidal dispersity, their active endocytosis form intestinal Payer's patches to increase oral bioavailability of rutin, making it a proper carrier for food fortification. Chelate formation is a result of interaction of rutin with metal ions. The chelation of metals can be crucial in the prevention of radical generation, which damages target biomolecules. Moreover, using natural chelators such as flavonoids is better than the synthetic ones due their toxicity effects. In the structure of several flavonoids (Fig1.0) are three potential coordination sites:

a) 5-hydroxy and 4-carbonyl group, b) 3-hydroxy and 4-carbonyl group, c) 3', 4'-hydroxy group in B ring. 

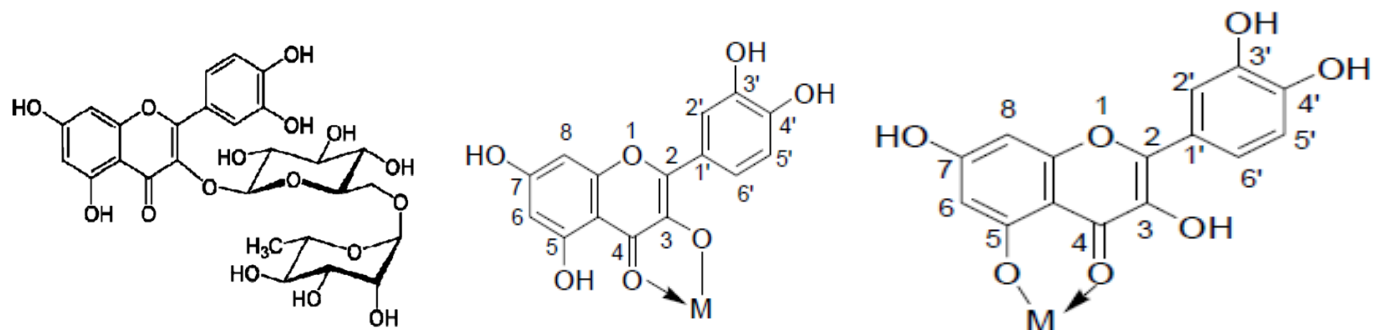

Figure 1. Structure of Rutin and Quarcitene. (M= Metal)

The interaction of certain flavonoids with transition metals increases their activity and leads to the formation of flavonoid-metal complexes, which may act as scavenger and free radical. Effect of serum albumin on stability of flavonoid-metal complexes was studied and complex of rutin with iron (II) was found to be the most stable. The ability of flavonoid metal complexes to catalyze homolytic cleavage of hydrogen peroxide was also studied and rutin iron (II) complex was found to be relatively poor Fenton catalyst. The potential therapeutic benefits of this new antioxidant agent were studied using experimental model of pathological states associated with oxidative stress in vivo. It was found that pretreatment with rutin- iron complex protected against thio-acetamide induced hepato-toxicity as observed by a significant reduction in the elevated levels of serum enzymes and partial normalization of GSH/GSSG ratio, glutathione peroxidase II and glutathione reductase activity in mice liver. The results demonstrate that flavonoid-metal complexes possess effective free radical scavenger ability and have potent therapeutic benefits for the treatment of oxidative stress-related diseases and dysfunction. The antioxidant activity of flavonoids may involve their ability to form complex body iron in non-redox-active (Baccan et al. (2012).

\section{Material Methods}

\section{Preparation of Metal Complexes of Rutin with Methanolic Extract of Citrus Peel Extract.}

\subsection{Synthesis of Iron Metal Complexes with Rutin}

Panhwar, Q. K., \& Memon, S. (2014). Methanolic solution of $\mathrm{FeNo}_{3} .9 \mathrm{H}_{2} \mathrm{O}(1 \mathrm{mmol})$ was added drop wise to a solution of Rutin $(2 \mathrm{mmol})$ in methanol $(10 \mathrm{~mL})$ and the mixture was stirred for $2.30 \mathrm{~h}$ at $80^{\circ} \mathrm{C}$. The mixture was cooled, and the precipitate was filtered off, washed with methanol and then air dried to obtained brown colored compounds.

\subsection{Synthesis of Copper Metal Complexes with Rutin}

Methanolic solution of $\mathrm{CuSO}_{4} .5 \mathrm{H}_{2} \mathrm{O} 10 \mathrm{~mL}(1 \mathrm{mmol})$ was added drop wise to a solution of rutin $(2 \mathrm{mmol})$ in methanol $(10 \mathrm{~mL})$ and the mixture was stirred for $15 \mathrm{~min}$ at $60^{\circ} \mathrm{C}$. The mixture was cooled, and the precipitate was filtered off, washed with methanol and then air dried to obtain blue green colored compounds.

\subsection{Synthesis of Zinc Metal Complexes with Rutin}

$10 \mathrm{~mL}$ Zinc sulphate $\left(\mathrm{ZnSO}_{4} .5 \mathrm{H}_{2} \mathrm{O}\right) \quad(1 \mathrm{mmol})$ methanolic solution was added drop wise to a solution of rutin $(2 \mathrm{mmol})$ and the mixture was stirred for $20 \mathrm{~min}$ at $60^{\circ} \mathrm{C}$. The mixture was cooled, and the precipitate was filtered off, washed with methanol and then air dried to obtain white colored compound.

\subsection{Synthesis of Nickel Metal Complexes with Rutin}

$10 \mathrm{~mL}$ methanolic solution of Nickel acetate $(\mathrm{Ni}$ $\left(\mathrm{CH}_{3} \mathrm{COO}\right)_{2} \cdot 4 \mathrm{H}_{2} \mathrm{O}(1 \mathrm{mmol})$ was added drop wise to a solution of rutin $(2 \mathrm{mmol})$ in methanol $(10 \mathrm{~mL})$ and the mixture was stirred for $4 \mathrm{~h}$ at $60^{\circ} \mathrm{C}$. The mixture was cooled, and the precipitate was filtered off, washed with methanol and then air dried to obtain green colored crystals.

\subsection{Synthesis of Molybdenum and Zirconium Metal Complexes with Rutin}

In two separate round bottom flasks, rutin $(0.664 \mathrm{~g}, 2$ mmol) was added to dissolve thoroughly till 15 minutes, then added zirconium nitrate $(0.215 \mathrm{~g}, 1 \mathrm{mmol})$ in one flask and sodium molybdate $(0.242 \mathrm{~g}, 1 \mathrm{mmol})$ in another flask under stirring containing rutin. First, the color of rutin solutions was lemon yellow; but it immediately turned deep orange after adding metal salts. After two hours of stirring at ambient temperature (Payan-Gome et al., 2010). The solutions were filtered to eliminate the unreacted part of the components used; the precipitates were collected, washed with small aliquot of diethyl ether and well dried over silica gel in vacuum desiccators. The color of solid complexes was found as burnt orange and medium violet red formed with overall yield of $72 \%$ and $60 \%$ for $\mathrm{Zr}^{4+}$ and $\mathrm{Mo}^{+6}$ complexes, respectively.

\section{Antioxidant Activity}

Antioxidants are widely used in dietary supplements and have been investigated for the prevention of diseases such as cancer and inflammatory diseases (Barku et al., 2013). 
In present study, the in vitro antioxidant activity of citrus peels was evaluated by DPPH radical scavenging method; reducing power assay and metal chelating activity compared to the standard viz., Gallic acid, ethylene-diamine-tetraacetate (EDTA), butylated hydroxyl toluene (BHT), catechin, ascorbic acid and citric acid.

\subsection{DPPH Radical Scavenging Activity}

DPPH (2, 2-diphenyl-1-picrylhydrazyl radical) scavenging activity was evaluated according to the method developed earlier and recently being followed by many researchers (Proestos et al., 2013). In brief different amounts of the tested sample $(50-250 \mu \mathrm{g} / \mathrm{mL})$ were added to $5 \mathrm{~mL}$ of a $0.004 \%$ methanol solution of DPPH. Finally, the absorbance was read against a blank at $517 \mathrm{~nm}$ after 30 min of incubation at room temperature. BHT was used as positive control while reaction mixture (DPPH radical solution) minus extract solution was taken as control.

Inhibition of free radical by DPPH in percent (IC \%) was calculated by using the equation.

$$
\text { IC } \%=\frac{\text { Control }- \text { Sample }}{\text { Control }} \times 100
$$

Percent inhibition concentration was plotted against concentrations and the standard curve was drawn using standard antioxidant to calculate the $\mathrm{IC}_{50}$ values for standard and different extracts. A lower $\mathrm{IC}_{50}$ value indicated more radical scavenging activity.

\subsection{Reducing Power Activity}

The reducing power assay of extracts/essential oils was determined by the method developed earlier with slight modifications (Sethi et al., 2015). In brief varying concentrations of tested sample were mixed with $2.5 \mathrm{~mL}$ of phosphate buffer $(200 \mathrm{mM}, \mathrm{pH}=6.6)$ and $2.5 \mathrm{~mL}$ of $1 \%$ $\mathrm{K}_{4}\left[\mathrm{Fe}(\mathrm{CN})_{6}\right]$. After incubation, $2.5 \mathrm{~mL}$ of $\mathrm{Cl}_{3} \mathrm{CCOOH}$ was added to the mixtures, followed by centrifugation at 650 rpm for $10 \mathrm{~min}$. The upper layer was mixed with $5 \mathrm{~mL}$ of distilled water and $1 \mathrm{~mL}$ of $0.1 \% \mathrm{FeCl}_{3}$. Absorbance of the resultant solutions was measured at $700 \mathrm{~nm}$ using Thermo Scientific (Evolution 201) UV spectrophotometer. The reducing power of samples was calculated by using the following formula:

$$
\mathrm{RP} \%=\frac{(\text { Control-Sample })}{\text { Control }} \times 100
$$

Percent inhibition of reducing power (RP \%) was plotted against concentrations and the standard curve was drawn using standard antioxidant (BHT) to calculate the $\mathrm{RP}_{50}$ values for standard and different extracts/EOs. The lower $\mathrm{RP}_{50}$ values indicated greater reducing power ability.

\subsection{Metal Chelating Activity}

The metal chelating activity of $\mathrm{Fe}^{2+}$ was examined by the methods, recently being practiced and based on the principle of the $\mathrm{Fe}^{2+}$ chelating ability the absorbance of ferrous iron-ferrozine complex formed was measured at $562 \mathrm{~nm}$ (Prakash et al., 2011). To execute the experiment $0.1 \mathrm{~mL}$ of $2 \mathrm{mM} \mathrm{FeCl}_{2} .4 \mathrm{H}_{2} \mathrm{O}, 0.2 \mathrm{~mL}$ of $5 \mathrm{mM}$ ferrozine and $4.7 \mathrm{~mL}$ of methanol was added to different concentrations of tested samples. After incubation, the absorbance of test samples was measured at $562 \mathrm{~nm}$. The metal chelating activity of citrus species expressed in percentage was calculated using the following formula:

$$
\text { IC } \%=\frac{(\text { Control-Sample })}{\text { Control }} \times 100
$$

The percent of chelating ability (IC \%) was plotted against concentrations and the standard curve was drawn using standard antioxidant (EDTA) to calculate the $\mathrm{IC}_{50}$ values.

\subsection{Superoxide Radical Scavenging Activity}

The effect of super oxide radical scavenging activity was determined by the nitroblue tetrazolium reduction method (Fu et al., 2010). All readings were taken in triplicate and ascorbic acid and quarcitine was used as the standard. The $\%$ inhibition was calculated by following equation.

$$
\text { IC } \%=\frac{(\text { Control- } \text { Sample })}{\text { Control }} \times 100
$$

Where, A0 was the absorbance of control and A1 was the absorbance of ethanolic extract or standard.

\section{Result and Discussion}

\section{Antioxidant activity}

Antioxidants are widely used in dietary supplements and it has been investigated for the prevention of diseases such as cancer and inflammatory diseases (Barku et al., 2013). In present study, the in vitro antioxidant activity of citrus peels was evaluated by DPPH radical scavenging method; reducing power assay and metal chelating activity compared to the standard viz., Gallic acid, ethylene-diamine-tetraacetate (EDTA), butylated hydroxyl toluene (BHT), catechin, ascorbic acid and citric acid.

\section{DPPH radical scavenging activity}

DPPH (2, 2-diphenyl-1-picrylhydrazyl radical) scavenging activity was evaluated according to the method developed earlier and recently being followed by many researchers (Proestos et al., 2013). Butylated hydroxyl toluene was used as positive control while reaction mixture (DPPH radical solution) minus extract solution was taken as control. Inhibition of free radical by DPPH in percent (IC \%) was calculated by using the equation. 


$$
\text { IC } \%=\frac{(\text { Control- Sample })}{\text { Control }} \times 100
$$

\section{Antioxidant activity of metal complexes and bioconjugate of citrus peel extract}

In present study, antioxidant activity of metal complexes of Rutin and bioconjugate of citrus peel extract was investigated using five different assays as follows.

\section{DPPH radical scavenging activity}

Mean percent DPPH radical scavenging activity of metal complexes of Rutin and bioconjugate of citrus peel extract (De Souza and De Giovani, 2004) in a dose depended manner with minimum at lower and maximum at higher dose level $(100 \mu \mathrm{g} / \mathrm{mL}-500 \mu \mathrm{g} / \mathrm{mL})$ are presented in Table 1 and Fig 2A. The radical scavenging potential of synthesized metal complexes in the form of their significant $(\mathrm{p}<0.05) \quad \mathrm{IC}_{50}$ values revealed the order as Rut-Zr $>$ Rut-Mo $>$ Rut-Zn $>$ Cit-Zn $>$ Cit-Mo $>$ Cit-Zr $>$ Rut-Fe $>$ Cit-Cu> Rut-Ni> Rut-Cu> Cit-Fe $>$ Cit-Ni (Table 2 Fig $2 \mathrm{~B}) \mathrm{IC}_{50}$ values of standards were found in the order as Rutin $\left(\mathrm{IC}_{50}=250\right)$.

Table 1. Percent DPPH radical scavenging activity of Metal complexes of Rutin and bioconjugate of citrus peel extract collected from Uttarakhand region at different concentration

\begin{tabular}{|c|c|c|c|c|c|c|}
\hline \multirow{2}{*}{ S.No. } & \multirow{2}{*}{$\begin{array}{c}\text { Metal } \\
\text { complexes }\end{array}$} & \multicolumn{5}{|c|}{ \% DPPH radical scavenging activity at $700 \mathrm{~nm}$. } \\
\hline & & $100 \mu \mathrm{g} / \mathrm{mL}$ & $200 \mu \mathrm{g} / \mathrm{mL}$ & $300 \mu \mathrm{g} / \mathrm{mL}$ & $400 \mu \mathrm{g} / \mathrm{mL}$ & $500 \mu \mathrm{g} / \mathrm{mL}$ \\
\hline 1 & Rut-Fe & $51.67 \pm 5.774$ & $65.15 \pm 6.198$ & $71 \pm 5.427$ & $75.45 \pm 0.508$ & $80.56 \pm 0.593$ \\
\hline 2 & Rut-Cu & $46.71 \pm 0.376$ & $54.35 \pm 0.052$ & $62.03 \pm 1.169$ & $78.22 \pm 0.076$ & $84.99 \pm 0.11$ \\
\hline 3 & Rut-Ni & $43.93 \pm 0.497$ & $62.37 \pm 0.444$ & $70.81 \pm 0.313$ & $76 \pm 10$ & $80.67 \pm 0.577$ \\
\hline 4 & Rut-Zn & $41.92 \pm 0.55$ & $62.46 \pm 1.746$ & $71.14 \pm 0.145$ & $76.22 \pm 3.755$ & $82.63 \pm 0.65$ \\
\hline 5 & Rut-Zr & $28.48 \pm 0.65$ & $32.22 \pm 0.554$ & $42.32 \pm 1.046$ & $67.95 \pm 0.734$ & $78.73 \pm 0.719$ \\
\hline 6 & Rut-Mo & $32.23 \pm 0.577$ & $46.84 \pm 0.755$ & $56.83 \pm 0.271$ & $65.11 \pm 0.526$ & $72.57 \pm 0.82$ \\
\hline 7 & Cit-Fe & $42.38 \pm 0$ & $50.39 \pm 0.439$ & $60.75 \pm 0.133$ & $70.32 \pm 0.306$ & $77.08 \pm 0.951$ \\
\hline 8 & Cit-Cu & $31 \pm 0.10$ & $45.37 \pm 0.641$ & $56.25 \pm 1.097$ & $64.62 \pm 1.764$ & $78.73 \pm 0.723$ \\
\hline 9 & Cit-Ni & $36.19 \pm 0.826$ & $60 \pm 11.962$ & $63.97 \pm 6.34$ & $68.41 \pm 1.198$ & $78.3 \pm 0.751$ \\
\hline 10 & Cit-Zn & $31.33 \pm 0.669$ & $43.4 \pm 0.52$ & $43.91 \pm 3.897$ & $55.33 \pm 0.577$ & $64.44 \pm 0.548$ \\
\hline 11 & Cit-Zr & $28.33 \pm 0.577$ & $44.22 \pm 0.697$ & $49.85 \pm 2.312$ & $54.78 \pm 1.344$ & $66.23 \pm 0.955$ \\
\hline 12 & Cit-Mo & $35.04 \pm 1.055$ & $46.82 \pm 0.635$ & $56.04 \pm 1.002$ & $64.6 \pm 1.249$ & $71.1 \pm 1.054$ \\
\hline 13 & Rut* & $36.33 \pm 1.528$ & $41 \pm 10$ & $52.33 \pm 2.517$ & $61.9 \pm 0.475$ & $71.48 \pm 0.289$ \\
\hline
\end{tabular}

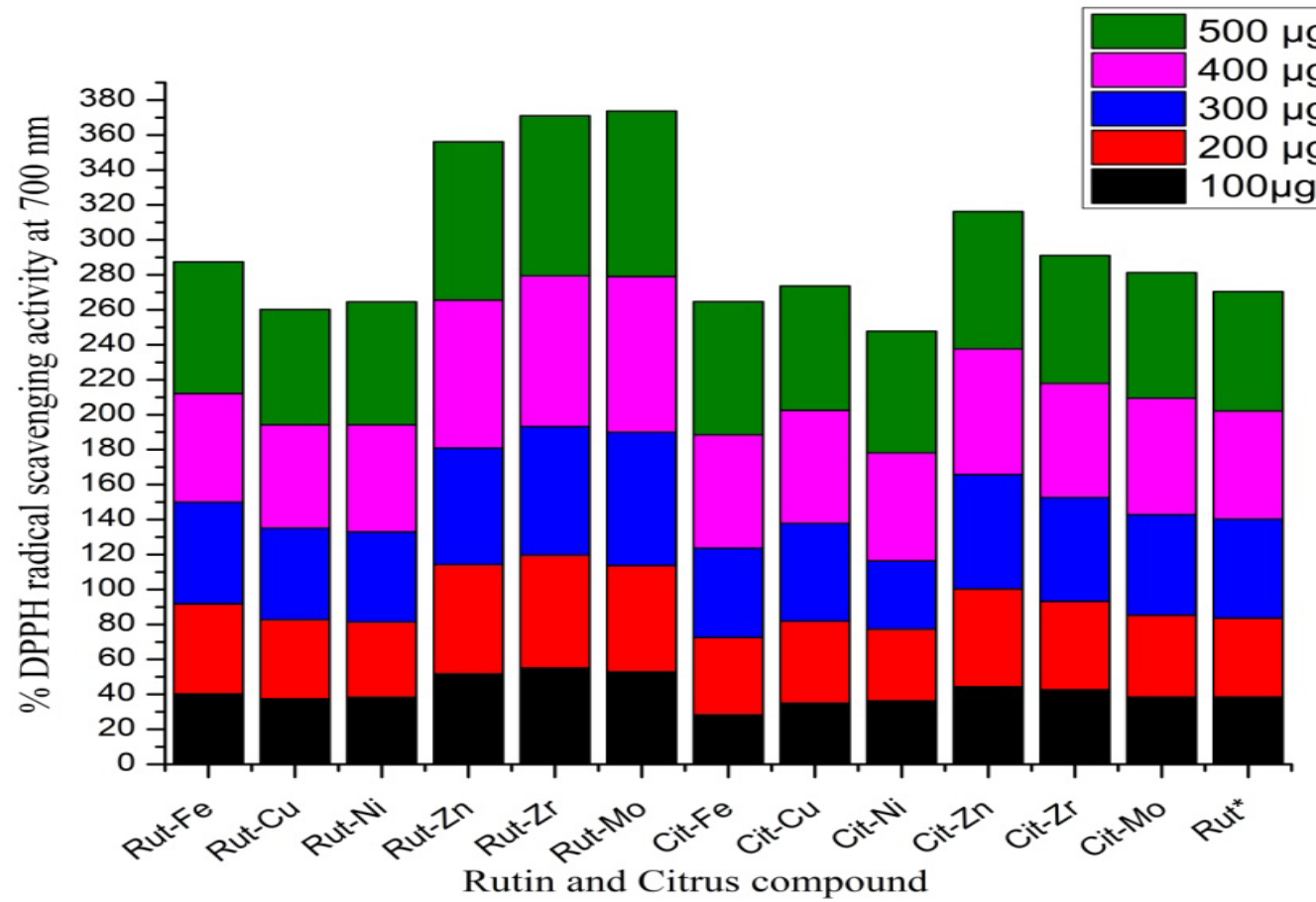

Figure 2A. Percent DPPH radical scavenging activity of metal complexes of Rutin and Bioconjugate of citrus peel extract collected from Uttarakhand region at different concentrations 
Table 2. $\mathrm{IC}_{50}$ of metal complexes of Rutin and bioconjugate of citrus peel extract for DPPH radical scavenging activity collected from Uttarakhand region at different concentrations

\begin{tabular}{|c|c|c|c|c|c|}
\hline \multirow{2}{*}{ S.No. } & \multirow{2}{*}{ Metal complexes } & \multicolumn{3}{|c|}{$\mathrm{IC}_{50}$ values $(\mu \mathrm{g} / \mathrm{mL})$ in triplicates } & \multirow{2}{*}{$\begin{array}{c}\text { Mean } \mathrm{IC}_{50} \text { values } \\
(\mu \mathrm{g} / \mathrm{mL})\end{array}$} \\
\hline & & $\mathrm{I}^{\mathrm{st}}$ & $\mathrm{II}^{\mathrm{nd}}$ & III $^{\text {rd }}$ & \\
\hline 1 & Rut-Fe & 24.41 & 24.34 & 23.22 & $23.99 \pm 0.668$ \\
\hline 2 & Rut-Cu & 107.81 & 108 & 108.11 & $107.97 \pm 0.152$ \\
\hline 3 & Rut-Ni & 148.7 & 149.31 & 148.11 & $148.71 \pm 0.6$ \\
\hline 4 & Rut-Zn & 122.94 & 122.98 & 123.32 & $123.08 \pm 0.209$ \\
\hline 5 & Rut-Zr & 300.95 & 300.1 & 300.17 & $300.41 \pm 0.472$ \\
\hline 6 & Rut-Mo & 252.22 & 256.33 & 326 & $278.18 \pm 41.461$ \\
\hline 7 & Cit-Fe & 186.74 & 181.23 & 181.4 & $183.12 \pm 3.133$ \\
\hline 8 & Cit-Cu & 256.31 & 257 & 258 & $257.1 \pm 0.85$ \\
\hline 9 & Cit-Ni & 178.47 & 179.99 & 180.11 & $179.52 \pm 0.914$ \\
\hline 10 & Cit-Zn & 330.38 & 330.23 & 330.45 & $330.35 \pm 0.112$ \\
\hline 11 & Cit-Zr & 316.62 & 320.45 & 319 & $318.69 \pm 1.934$ \\
\hline 12 & Cit-Mo & 250 & 250 & 252.11 & $250.7 \pm 1.218$ \\
\hline 13 & Rut* & 272.08 & 275.55 & 276 & $274.54 \pm 2.145$ \\
\hline
\end{tabular}

Values are mean of three replicates \pm Standard deviation. Within column, mean values followed by the same letter are not significantly different according to Tukey's test $(\mathrm{p}<0.05)$.

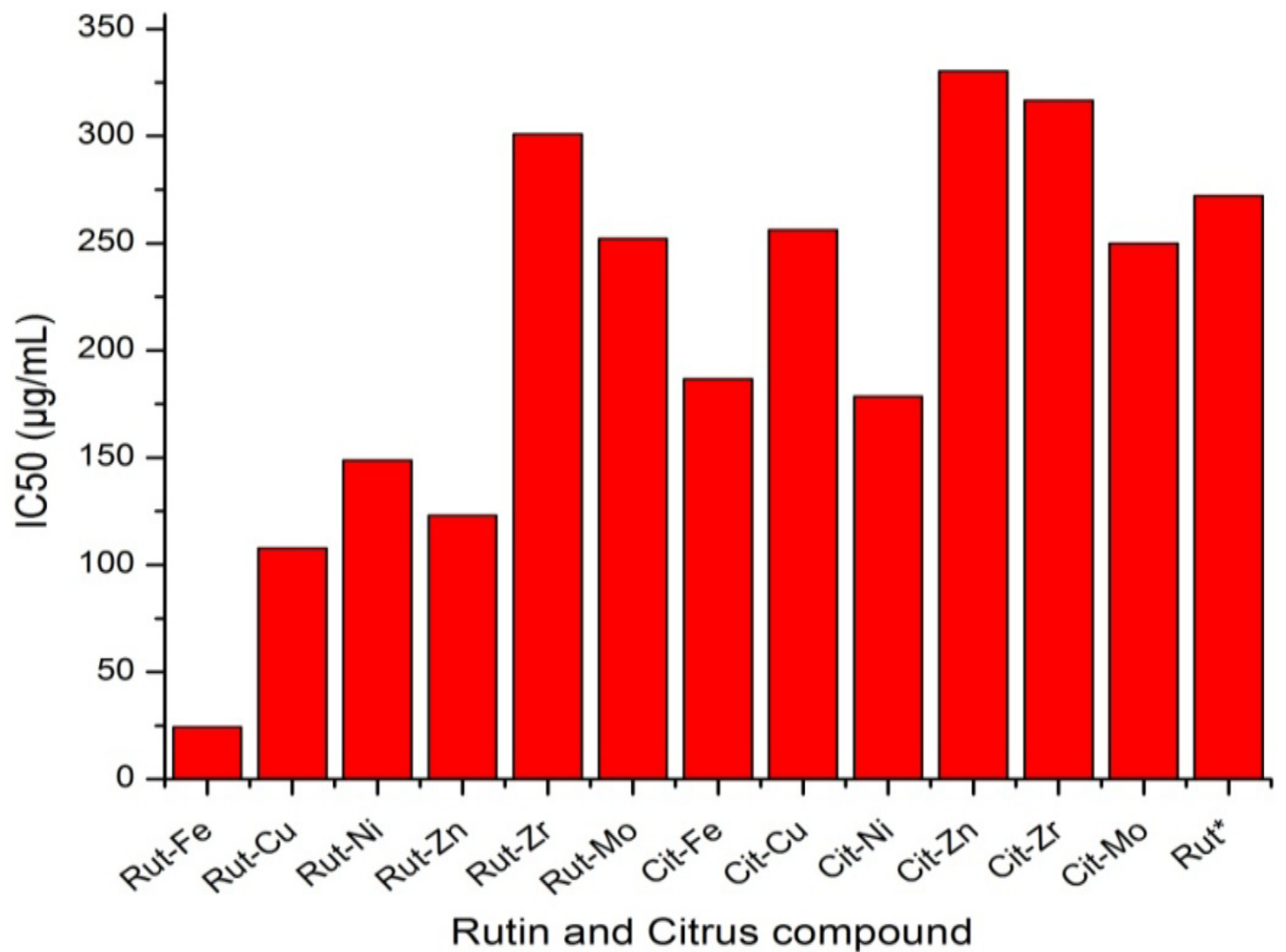

Figure 2B. $\mathrm{IC}_{50}$ of DPPH activity of citrus accession collected from Uttarakhand at different concentration 


\section{Hydroxyl radical scavenging activity}

Table 3 and Fig 2C Presented mean percent hydroxyl radical scavenging activity of metal complexes of Rutin and bioconjugate of citrus peel extract in a dose depended manner with minimum at lower and maximum at higher dose level $(100 \mu \mathrm{g} / \mathrm{mL}-500 \mu \mathrm{g} / \mathrm{mL})$. $\mathrm{IC}_{50}(\mathrm{p}<0.05)$ values of synthesized metal complexes for hydroxyl radical scavenging activity exhibited the order as Rut-Fe $>$ Rut-Mo $>$ Cit-Ni $>$ Cit-Fe $>$ Rut-Zn $>$ Cit-Mo $>$ Cit-Cu $>$ Cit-Cu $>$ Rut-Zr $>$ Cit-Zr $>$ Rut-Ni $>$ Rut-Cu $>$ Cit-Zn (Table 4 and Fig 2D) $\mathrm{IC}_{50}$ values of standards were found in the order as Rutin $\left(\mathrm{IC}_{50}=169.72\right)$.

Table 3. Percent Hydroxyl radical scavenging activity of citrus peel extract collected from Uttarakhand region at different concentration

\begin{tabular}{|c|c|c|c|c|c|c|}
\hline \multirow{2}{*}{ S.No } & \multirow{2}{*}{ Metal complexes } & \multicolumn{5}{|c|}{ \% Hydroxyl radical scavenging activity at $\mathbf{5 6 0} \mathbf{~ n m}}$. \\
\cline { 3 - 7 } & & $\mathbf{1 0 0} \mu \mathbf{g} / \mathbf{m L}$ & $\mathbf{2 0 0} \boldsymbol{\mu} \mathbf{g} \mathbf{m L}$ & $\mathbf{3 0 0} \boldsymbol{\mu \mathbf { g } / \mathbf { m L }}$ & $\mathbf{4 0 0} \boldsymbol{\mu g} / \mathbf{m L}$ & $\mathbf{5 0 0} \boldsymbol{\mu g} / \mathbf{m L}$ \\
\hline 1 & Rut-Fe & $53.57 \pm 1.326$ & $53.97 \pm 0.728$ & $67.32 \pm 0.812$ & $71.08 \pm 0.052$ & $84.46 \pm 0.098$ \\
\hline 2 & Rut-Cu & $34 \pm 1.732$ & $43.33 \pm 0.577$ & $51.17 \pm 1.607$ & $61.95 \pm 2.531$ & $69.8 \pm 1.323$ \\
\hline 3 & Rut-Ni & $32.15 \pm 1.117$ & $44.41 \pm 0.514$ & $56.3 \pm 0.624$ & $63.4 \pm 1.253$ & $70.23 \pm 0.208$ \\
\hline 4 & Rut-Zn & $41.25 \pm 0.718$ & $61.13 \pm 1.588$ & $51.14 \pm 0.145$ & $65.56 \pm 3.246$ & $72.63 \pm 0.650$ \\
\hline 5 & Rut-Zr & $36.73 \pm 1.861$ & $47.37 \pm 0.641$ & $61.33 \pm 1.258$ & $65.94 \pm 0.907$ & $73.4 \pm 1.219$ \\
\hline 6 & Rut-Mo & $41.6 \pm 0.520$ & $56.51 \pm 0.277$ & $66.83 \pm 0.271$ & $67.78 \pm 0.728$ & $68.57 \pm 0.82$ \\
\hline 7 & Cit-Fe & $40.38 \pm 0$ & $57.73 \pm 1.016$ & $65.08 \pm 2.754$ & $66.98 \pm 0.271$ & $76.75 \pm 1.174$ \\
\hline 8 & Cit-Cu & $42.94 \pm 1.330$ & $49.89 \pm 0.023$ & $56.16 \pm 0.706$ & $67.62 \pm 0.826$ & $78.73 \pm 0.723$ \\
\hline 9 & Cit-Ni & $46.19 \pm 0.826$ & $52 \pm 3.721$ & $60.3 \pm 0.318$ & $68.41 \pm 1.198$ & $70.64 \pm 0.271$ \\
\hline 10 & Cit-Zn & $23.45 \pm 0.672$ & $34.67 \pm 1.528$ & $39 \pm 1$ & $52.67 \pm 1.528$ & $64.67 \pm 1.528$ \\
\hline 11 & Cit-Zr & $36.3 \pm 1.113$ & $41.78 \pm 1.603$ & $59.36 \pm 0.271$ & $61.11 \pm 0.277$ & $71.97 \pm 0.058$ \\
\hline 12 & Cit-Mo & $43 \pm 1$ & $49 \pm 1$ & $59.52 \pm 0$ & $61.9 \pm 0.475$ & $63.81 \pm 0.48$ \\
\hline 13 & Rut* & $44 \pm 1$ & $53.37 \pm 0.548$ & $61 \pm 1$ & $75.1 \pm 0.458$ & $87.67 \pm 1.528$ \\
\hline
\end{tabular}

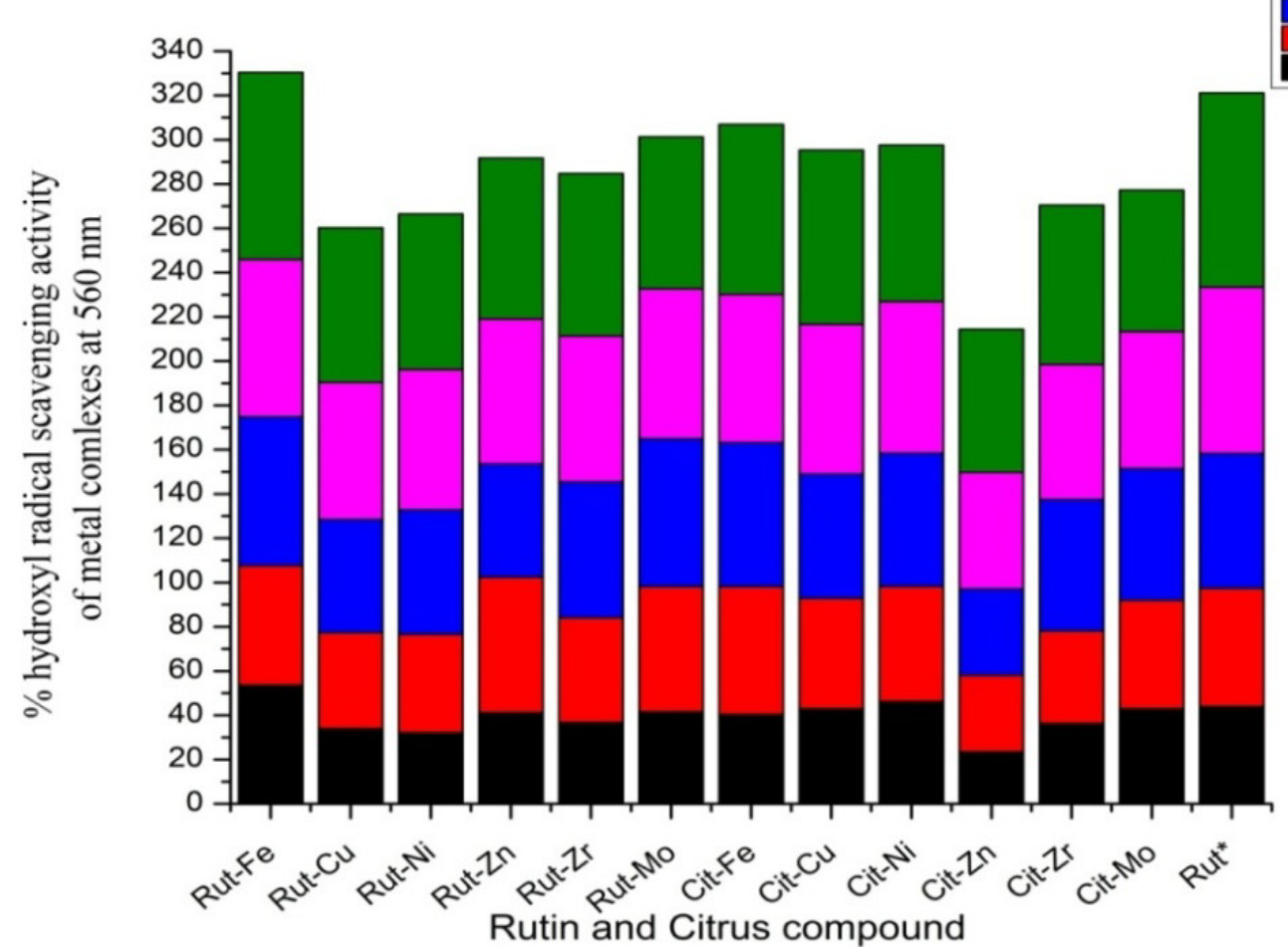

Figure 2C. Percent Hydroxyl radical scavenging activity of metal complexes of Rutin and Bioconjugate of citrus peel extract collected from Uttarakhand region at different concentrations 
Table 4. $\mathrm{IC}_{50}$ of metal complexes of Rutin and bioconjugate of citrus peel extract for Hydroxyl radical scavenging activity collected from Uttarakhand region at different concentrations

\begin{tabular}{|c|c|c|c|c|c|}
\hline \multirow{2}{*}{ S.No. } & \multirow{2}{*}{ Metal complexes } & \multicolumn{3}{|c|}{ IC $_{50}$ values $(\mu \mathrm{g} / \mathrm{mL})$ in triplicates } & \multirow{2}{*}{$\begin{array}{c}\text { Mean } \text { IC }_{50} \text { values } \\
(\mu \mathrm{g} / \mathbf{m} \mathbf{L})\end{array}$} \\
\hline & & $I^{\text {st }}$ & II $^{\text {nd }}$ & III $^{\text {rd }}$ & \\
\hline 1 & Rut-Fe & 97.3 & 97.3 & 96.9 & $97.17 \pm 0.231^{\mathrm{m}}$ \\
\hline $8 \mathrm{u} 2$ & Rut-Cu & 278 & 277.44 & 276 & $277.15 \pm 1.032^{b}$ \\
\hline 3 & Rut-Ni & 265 & 263.5 & 264.5 & $264.33 \pm 0.764^{\mathrm{c}}$ \\
\hline 4 & Rut-Zn & 179 & 179.78 & 178 & $178.93 \pm 0.892^{\mathrm{h}}$ \\
\hline 5 & Rut-Zr & 226.59 & 225.55 & 224.5 & $225.55 \pm 1.045^{\mathrm{e}}$ \\
\hline 6 & Rut-Mo & 143.23 & 144.1 & 143.23 & $143.52 \pm 0.502^{1}$ \\
\hline 7 & Cit-Fe & 161.21 & 160 & 161.11 & $160.77 \pm 0.672^{\mathrm{j}}$ \\
\hline 8 & Cit-Cu & 199.21 & 199.21 & 198.34 & $198.92 \pm 0.502^{\mathrm{g}}$ \\
\hline 9 & Cit-Ni & 155.23 & 155.23 & 155.35 & $155.27 \pm 0.069^{\mathrm{k}}$ \\
\hline 10 & Cit-Zn & 372.4 & 373.341 & 375 & $373.58 \pm 1.316^{\mathrm{a}}$ \\
\hline 11 & Cit-Zr & 256.66 & 256 & 255.5 & $256.05 \pm 0.582^{\mathrm{d}}$ \\
\hline 12 & Cit-Mo & 202.3 & 205.92 & 205 & $204.41 \pm 1.882^{\mathrm{f}}$ \\
\hline 13 & Rut* & 169.72 & 169.2 & 169.4 & $169.440 .262^{\mathrm{i}}$ \\
\hline
\end{tabular}

Values are mean of three replicates \pm Standard deviation. Within column, mean values followed by the same letter are not significantly different according to Tukey's test $(\mathrm{p}<0.05)$.

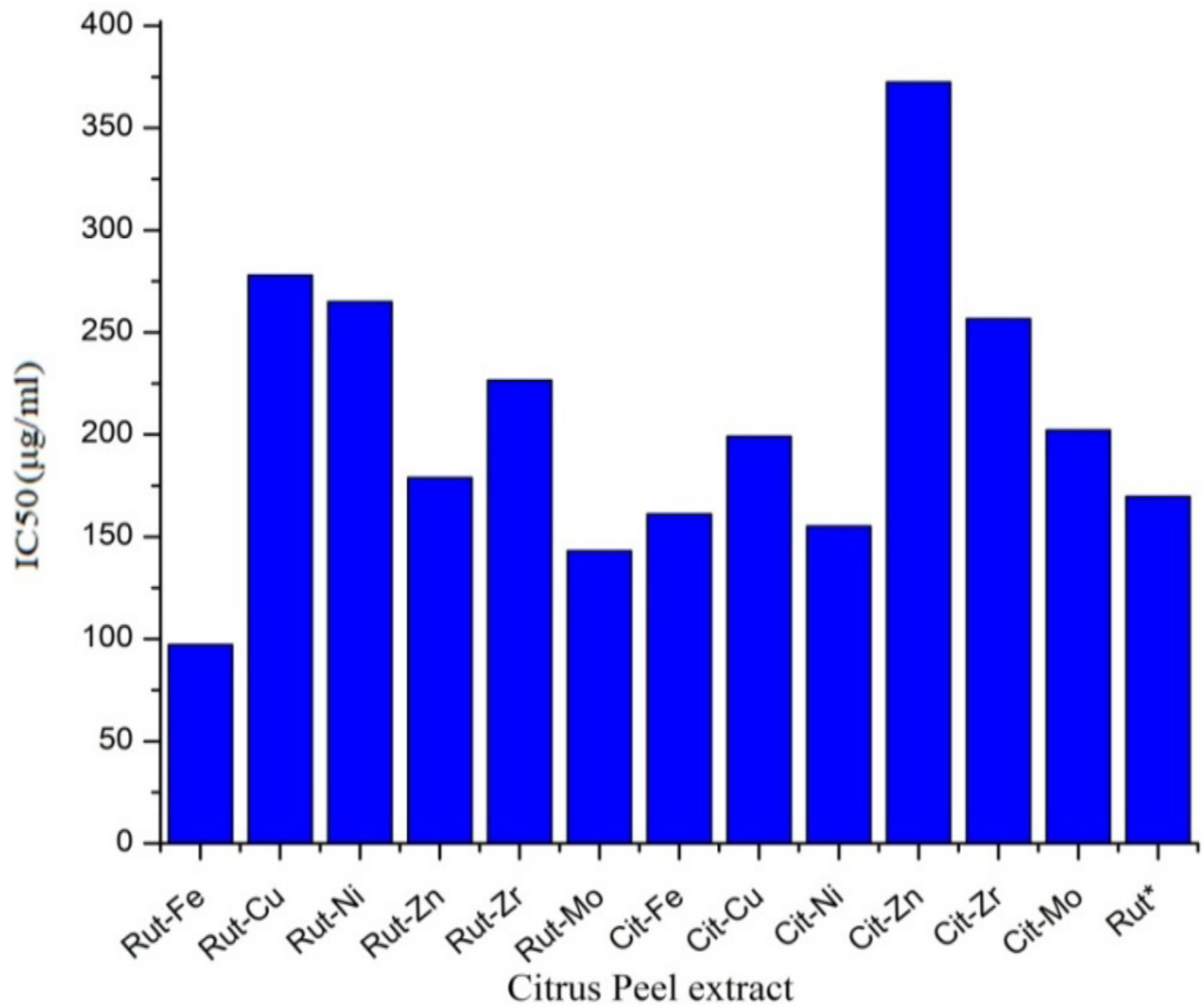

Figure 2D. $\mathrm{IC}_{50}$ of Metal complexes of Rutin and bioconjugate of citrus peel extract collected from Uttarakhand region for Hydroxyl radical inhibition scavenging activity 


\section{Superoxide anion scavenging activity}

Mean percent superoxide anion scavenging activity of metal complexes of Rutin and bioconjugate of citrus peel extract in a dose depended manner with minimum at lower and maximum at higher dose level $(100 \mu \mathrm{g} / \mathrm{mL}-500 \mu \mathrm{g} / \mathrm{mL})$ are presented in Table 5and fig $2 \mathrm{E}$. The superoxide anion scavenging potential of synthesized metal complexes in the form of their significant $(\mathrm{p}<0.05), \mathrm{IC}_{50}$ values revealed the order as Rut-Fe $>$ Cit-Zn $>$ Cit-Fe $>$ Cit-Fe $>$ Rut-Zn $>$ Cit-Ni $>$ Cit-Mo $>$ Rut-Zr $>$ Cit-Zr $>$ Rut-Mo $>$ Rut-Ni $>$ Rut-Cu Table 6 and Fig $2 \mathrm{~F}$ the IC50 values of standards were found in the order as Rutin $\left(\mathrm{IC}_{50}=250\right)$.

Table 5. Percent superoxide radical anion scavenging activity of citrus peel extract collected from different region of Uttarakhand at different concentration

\begin{tabular}{|c|c|c|c|c|c|c|}
\hline \multirow{2}{*}{ S.No } & \multirow{2}{*}{ Metal complexes } & \multicolumn{5}{|c|}{ \% superoxide radical anion scavenging activity at 560 $\mathbf{~ m}}$. \\
\cline { 3 - 7 } & & $\mathbf{1 0 0} \mu \mathbf{g} / \mathbf{m L}$ & $\mathbf{2 0 0} \boldsymbol{\mu} \mathbf{g} \mathbf{m L}$ & $\mathbf{3 0 0} \boldsymbol{\mu} \mathbf{g} \mathbf{m L}$ & $\mathbf{4 0 0} \boldsymbol{\mu g} / \mathbf{m L}$ & $\mathbf{5 0 0} \boldsymbol{\mu g} / \mathbf{M I}$ \\
\hline 1 & Rut-Fe & $41.04 \pm 1.12$ & $50.7 \pm 0.936$ & $67.32 \pm 0.812$ & $71.75 \pm 1.207$ & $84.46 \pm 0.098$ \\
\hline 2 & Rut-Cu & $24.19 \pm 1.612$ & $34.52 \pm 1.775$ & $38.45 \pm 1.018$ & $42 \pm 1$ & $66.33 \pm 0.577$ \\
\hline 3 & Rut-Ni & $25.93 \pm 0.586$ & $38.48 \pm 0.501$ & $44.34 \pm 1.658$ & $55.45 \pm 0.508$ & $70.56 \pm 0.593$ \\
\hline 4 & Rut-Zn & $36.71 \pm 0.376$ & $44.35 \pm 0.052$ & $52.03 \pm 1.169$ & $65.22 \pm 0.076$ & $72.65 \pm 1.045$ \\
\hline 5 & Rut-Zr & $33.93 \pm 0.497$ & $42.37 \pm 0.444$ & $50.81 \pm 0.313$ & $56 \pm 1$ & $75.74 \pm 1.512$ \\
\hline 6 & Rut-Mo & $38.41 \pm 0.271$ & $47.19 \pm 0.287$ & $56.76 \pm 0.287$ & $61.74 \pm 0.271$ & $68.38 \pm 0$ \\
\hline 7 & Cit-Fe & $18.48 \pm 0.65$ & $34.89 \pm 1.755$ & $42.32 \pm 1.046$ & $57.95 \pm 0.734$ & $78.4 \pm 0.252$ \\
\hline 8 & Cit-Cu & $42.54 \pm 0.277$ & $50.6 \pm 0.271$ & $59.52 \pm 0$ & $65.24 \pm 0.745$ & $73.24 \pm 1.076$ \\
\hline 9 & Cit-Ni & $42.38 \pm 0$ & $50.39 \pm 0.439$ & $60.75 \pm 0.133$ & $70.32 \pm 0.306$ & $77.08 \pm 0.951$ \\
\hline 10 & Cit-Zn & $31 \pm 1$ & $45.37 \pm 0.641$ & $56.25 \pm 1.097$ & $64.62 \pm 1.764$ & $78.73 \pm 0.723$ \\
\hline 11 & Cit-Zr & $36.19 \pm 0.826$ & $60 \pm 11.962$ & $63.97 \pm 6.34$ & $68.41 \pm 1.198$ & $78.3 \pm 0.751$ \\
\hline 12 & Cit-Mo & $31.33 \pm 0.669$ & $43.4 \pm 0.52$ & $43.91 \pm 3.897$ & $55.33 \pm 0.577$ & $68.78 \pm 0.029$ \\
\hline 13 & Rut* & $28.33 \pm 0.577$ & $44.22 \pm 0.697$ & $51.15 \pm 1.227$ & $64.78 \pm 1.344$ & $76.23 \pm 0.955$ \\
\hline
\end{tabular}

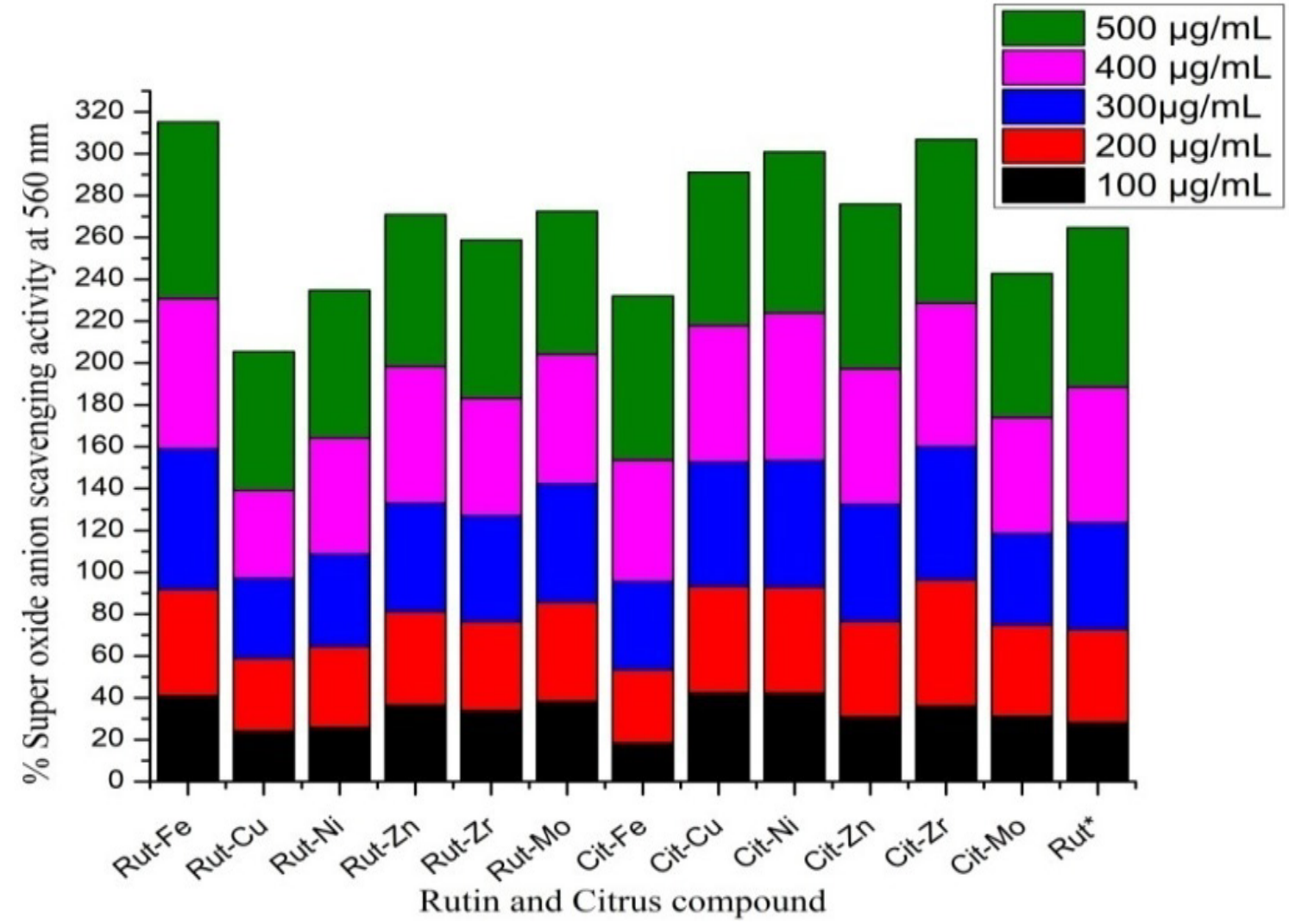

Figure 2E. Percent superoxide anion scavenging activity of metal complexes of Rutin and Bioconjugate of citrus peel extract collected from Uttarakhand region at different concentrations 
Table 6. $\mathrm{IC}_{50}$ of Metal complexes of Rutin and citrus peel extract for superoxide radical scavenging activity collected from Uttarakhand region at different concentrations

\begin{tabular}{|c|c|c|c|c|c|}
\hline \multirow{2}{*}{ S.No } & \multirow{2}{*}{ Metal complexes } & \multicolumn{3}{|c|}{ IC $_{50}$ values $(\mu \mathrm{g} / \mathrm{mL})$ in triplicates } & \multirow{2}{*}{$\begin{array}{c}\text { Mean } \text { IC }_{50} \text { values } \\
(\mu \mathrm{g} / \mathrm{mL})\end{array}$} \\
\hline & & $I^{\text {st }}$ & II $^{\text {nd }}$ & III $^{\text {rd }}$ & \\
\hline 1 & Rut-Fe & 180 & 180.34 & 181.34 & $180.56 \pm 0.697$ \\
\hline 2 & Rut-Cu & 400.32 & 400 & 399.91 & $400.08 \pm 0.215$ \\
\hline 3 & Rut-Ni & 329.43 & 328.44 & 227 & $294.96 \pm 58.854$ \\
\hline 4 & Rut-Zn & 256.43 & 256.44 & 255 & $255.96 \pm 0.829$ \\
\hline 5 & Rut-Zr & 282.57 & 280 & 280 & $280.86 \pm 1.484$ \\
\hline 6 & Rut-Mo & 327.19 & 326.33 & 326 & $326.51 \pm 0.614$ \\
\hline 7 & Cit-Fe & 191.97 & 191.23 & 191.4 & $191.53 \pm 0.388$ \\
\hline 8 & Cit-Cu & 186.74 & 185 & 184.6 & $185.45 \pm 1.138$ \\
\hline 9 & Cit-Ni & 256.31 & 255 & 258.34 & $256.55 \pm 1.683$ \\
\hline 10 & Cit-Zn & 178.42 & 180.33 & 185.34 & $181.36 \pm 3.574$ \\
\hline 11 & Cit-Zr & 319.76 & 320.45 & 319 & $319.74 \pm 0.725$ \\
\hline 12 & Cit-Mo & 275.6 & 280 & 278.11 & $277.9 \pm 2.207$ \\
\hline 13 & Rut* & 306.17 & 305.55 & 306 & $305.91 \pm 0.32$ \\
\hline
\end{tabular}

Values are mean of three replicates \pm Standard deviation. Within column, mean values followed by the same letter are not significantly different according to Tukey's test $(\mathrm{p}<0.05)$.

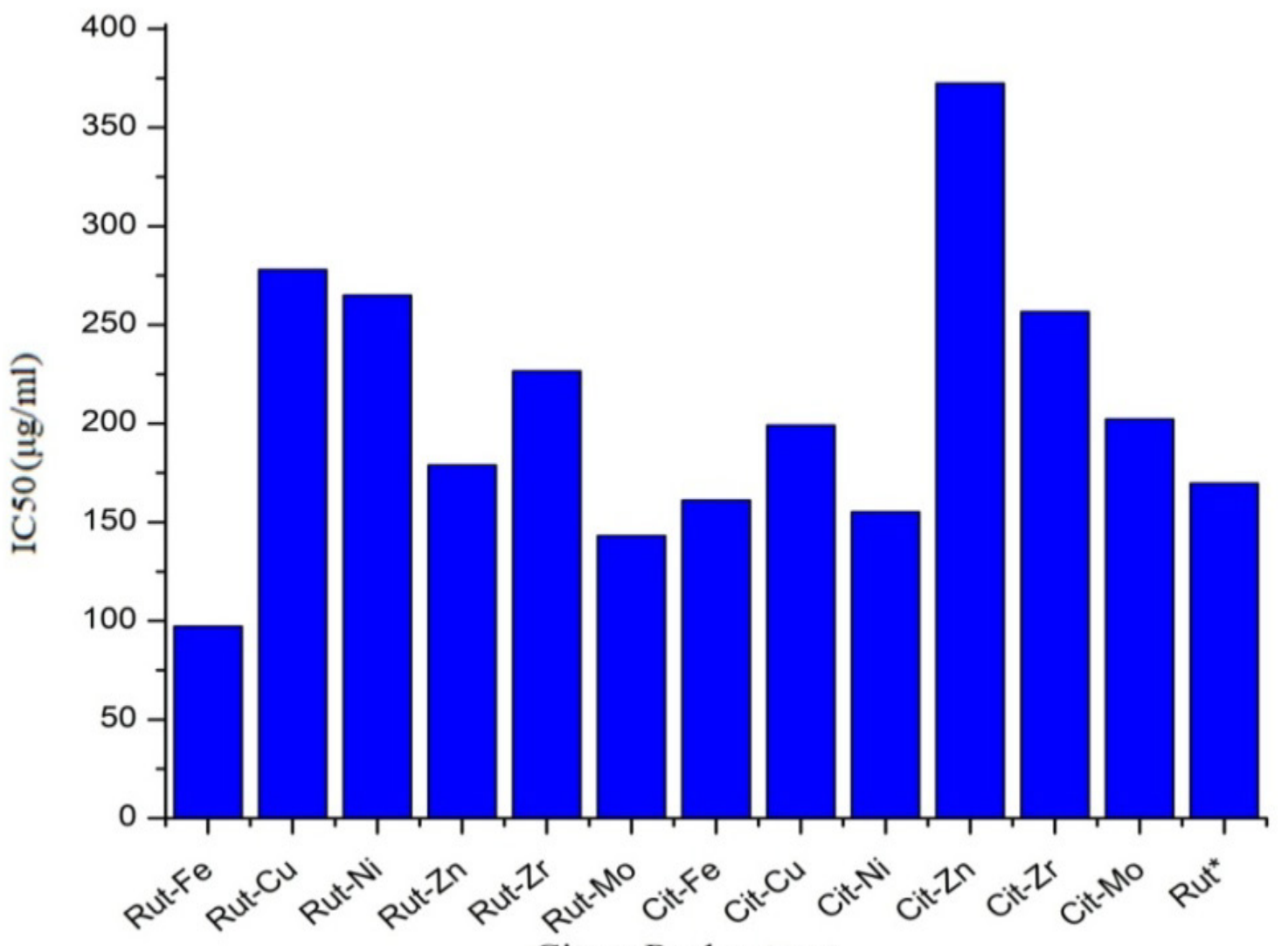

Citrus Peel extract

Figure 2F. $\mathrm{IC}_{50}$ of Metal complexes of Rutin and bioconjugate of citrus peel extract collected from Uttarakhand region for Superoxide radical inhibition scavenging activity 


\section{Metal chelating activity}

Table 7 and Fig 2G presented mean percent metal chelating activity of metal complexes of Rutin and bioconjugate of citrus peel extract in a dose depended manner with minimum at lower and maximum at higher dose level $(100 \mu \mathrm{g} / \mathrm{mL}-500 \mu \mathrm{g} / \mathrm{mL})$. $\mathrm{IC}_{50}(\mathrm{p}<0.05)$ values of synthesized metal complexes for metal chelating activity exhibited the order as Rut-Zr $>$ Rut- $\mathrm{Cu}>$ Rut-Zn>Rut-Fe $>$ Cit-Zr $>\quad$ Cit-Ni $>$ Rut-Ni $>$ Rut-Mo $>$ Cit-Zn $>$ Cit-Mo $>$ Cit-Fe $>$ Cit-Cu (Table 8 Fig $2 \mathrm{H}) \mathrm{IC}_{50}$ values of standards were found in the order as Rutin $\left(\mathrm{IC}_{50}=250\right)$.

Table 7. Percent Metal chelating activity of metal complexes of Rutin and Bioconjugate of citrus peel extract collected from Uttarakhand region at different concentrations

\begin{tabular}{|c|c|c|c|c|c|c|}
\hline \multirow{2}{*}{ S.No. } & \multirow{2}{*}{ Metal complexes } & \multicolumn{5}{|c|}{ \% Metal chelating activity at 560 nm. } \\
\cline { 3 - 7 } & & $\mathbf{1 0 0} \mu \mathbf{g} / \mathbf{m L}$ & $\mathbf{2 0 0} \boldsymbol{\mu} \mathbf{g} \mathbf{m L}$ & $\mathbf{3 0 0} \boldsymbol{\mu \mathbf { g } / \mathbf { m L }}$ & $\mathbf{4 0 0} \boldsymbol{\mu \mathbf { g }} / \mathbf{m L}$ & $\mathbf{5 0 0} \boldsymbol{\mu g} / \mathbf{m L}$ \\
\hline 1 & Rut-Fe & $44.37 \pm 6.894$ & $50.7 \pm 0.936$ & $67.32 \pm 0.812$ & $71.08 \pm 0.052$ & $84.46 \pm 0.098$ \\
\hline 2 & Rut-Cu & $44.19 \pm 2.645$ & $54.52 \pm 1.775$ & $66.45 \pm 3.423$ & $71 \pm 1$ & $76.33 \pm 0.577$ \\
\hline 3 & Rut-Ni & $35 \pm 0$ & $48.48 \pm 0.501$ & $54.34 \pm 1.658$ & $65.45 \pm 0.508$ & $70.56 \pm 0.593$ \\
\hline 4 & Rut-Zn & $46.71 \pm 0.376$ & $54.35 \pm 0.052$ & $62.03 \pm 1.169$ & $78.22 \pm 0.076$ & $84.99 \pm 0.11$ \\
\hline 5 & Rut-Zr & $43.93 \pm 0.497$ & $62.37 \pm 0.444$ & $70.81 \pm 0.313$ & $76 \pm 1$ & $80.67 \pm 0.577$ \\
\hline 6 & Rut-Mo & $31.92 \pm 0.55$ & $43.13 \pm 1.038$ & $51.14 \pm 0.145$ & $68.22 \pm 0.4$ & $72.63 \pm 0.65$ \\
\hline 7 & Cit-Fe & $28.48 \pm 0.65$ & $34.89 \pm 1.755$ & $42.32 \pm 1.046$ & $57.95 \pm 0.734$ & $68.73 \pm 0.719$ \\
\hline 8 & Cit-Cu & $22.23 \pm 0.577$ & $36.51 \pm 0.277$ & $46.83 \pm 0.271$ & $55.11 \pm 0.526$ & $62.57 \pm 0.82$ \\
\hline 9 & Cit-Ni & $42.38 \pm 0$ & $50.39 \pm 0.439$ & $60.75 \pm 0.133$ & $70.32 \pm 0.306$ & $77.08 \pm 0.951$ \\
\hline 10 & Cit-Zn & $31 \pm 1.0$ & $45.37 \pm 0.641$ & $56.25 \pm 1.097$ & $64.62 \pm 1.764$ & $78.73 \pm 0.723$ \\
\hline 11 & Cit-Zr & $36.19 \pm 0.826$ & $60 \pm 11.962$ & $63.97 \pm 6.34$ & $68.41 \pm 1.198$ & $78.3 \pm 0.751$ \\
\hline 12 & Cit-Mo & $31.33 \pm 0.669$ & $43.4 \pm 0.52$ & $43.91 \pm 3.897$ & $55.33 \pm 0.577$ & $64.44 \pm 0.548$ \\
\hline 13 & Rut* & $28.33 \pm 0.577$ & $44.22 \pm 0.697$ & $51.15 \pm 1.227$ & $64.78 \pm 1.344$ & $76.23 \pm 0.955$ \\
\hline
\end{tabular}

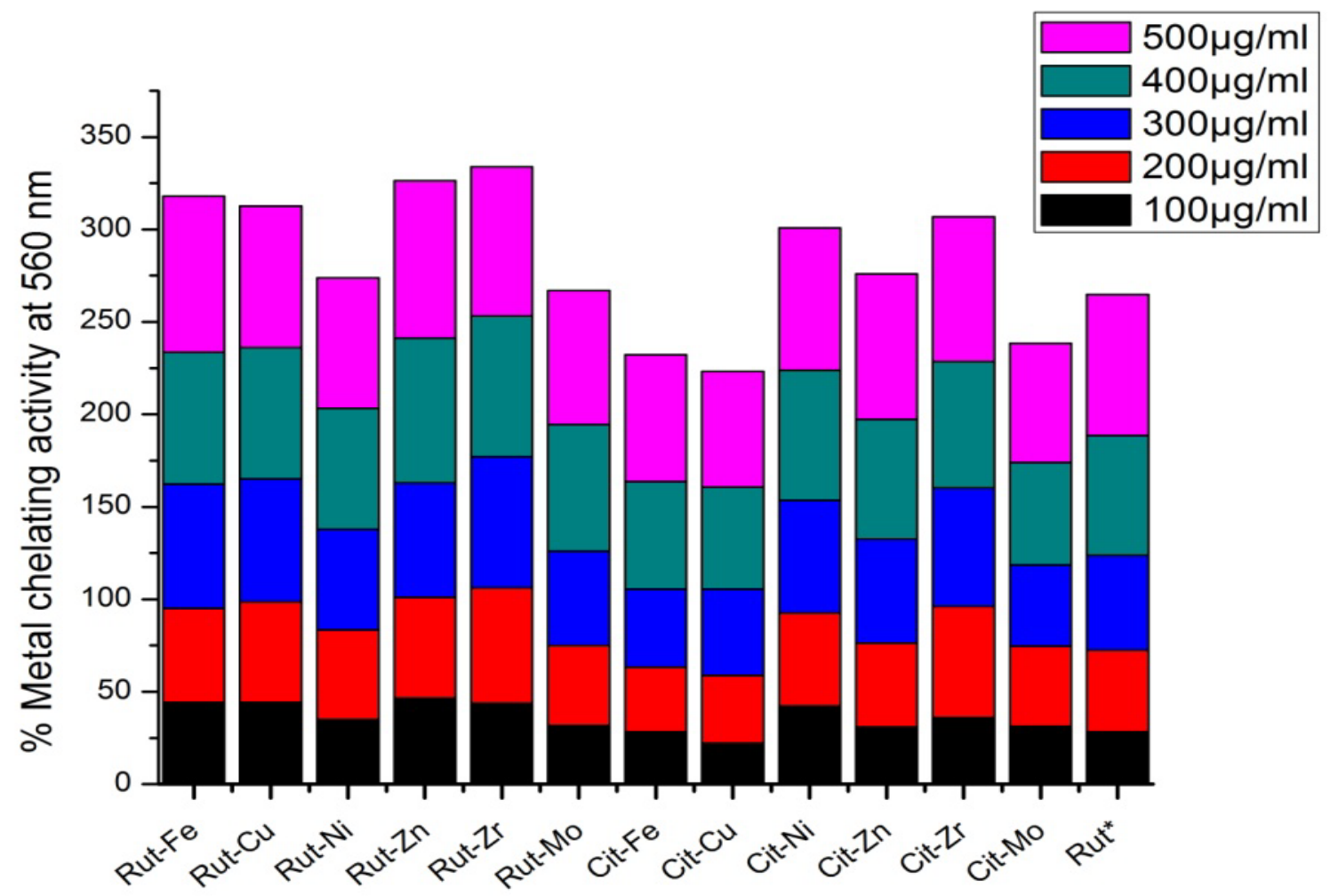

Figure 2G. Percent Metal chelating activity of metal complexes of Rutin and Bioconjugate of citrus peel extract collected from Uttarakhand region at different concentrations 
Table 8. $\mathrm{IC}_{50}$ of Metal complexes of rutin and bioconjugate of citrus peel extract for Metal chelating activity collected from Uttarakhand region at different concentrations

\begin{tabular}{|c|c|c|c|c|c|}
\hline \multirow{2}{*}{ S.No } & \multirow{2}{*}{ Metal complex } & \multicolumn{3}{|c|}{ Mean $\mathrm{IC}_{50}$ values $(\mu \mathrm{g} / \mathrm{mL})$ in triplicates } & \multirow{2}{*}{$\begin{array}{c}\text { Mean } \text { IC }_{50} \text { values } \\
(\mu \mathrm{g} / \mathrm{mL})\end{array}$} \\
\hline & & I $^{\text {st }}$ & II $^{\text {nd }}$ & III $^{\text {rd }}$ & \\
\hline 1 & Rut-Fe & 165.9 & 164.34 & 163.22 & $164.49 \pm 1.346$ \\
\hline 2 & Rut-Cu & 146.625 & 148 & 148.11 & $147.58 \pm 0.827$ \\
\hline 3 & Rut-Ni & 246.25 & 249.31 & 248.11 & $247.89 \pm 1.542$ \\
\hline 4 & Rut-Zn & 148.7 & 148.98 & 145.32 & $147.67 \pm 2.037$ \\
\hline 5 & Rut-Zr & 107.81 & 100.1 & 105.17 & $104.36 \pm 3.918$ \\
\hline 6 & Rut-Mo & 269.33 & 266.33 & 266 & $267.22 \pm 1.835$ \\
\hline 7 & Cit-Fe & 335.92 & 335.23 & 333.4 & $334.85 \pm 1.302$ \\
\hline 8 & Cit-Cu & 354.94 & 257 & 258 & $289.98 \pm 56.259$ \\
\hline 9 & Cit-Ni & 186.74 & 179.99 & 180.11 & $182.28 \pm 3.863$ \\
\hline 10 & Cit-Zn & 256.31 & 250.23 & 250.45 & $252.33 \pm 3.449$ \\
\hline 11 & Cit-Zr & 178.47 & 180.45 & 180 & $179.64 \pm 1.038$ \\
\hline 12 & Cit-Mo & 330.38 & 330 & 332.11 & $330.83 \pm 1.125$ \\
\hline 13 & Rut* & 275.6 & 275.55 & 276 & $275.72 \pm 0.247$ \\
\hline
\end{tabular}

Values are mean of three replicates \pm Standard deviation. Within column, mean values followed by the same letter are not significantly different according to Tukey's test $(\mathrm{p}<0.05)$.

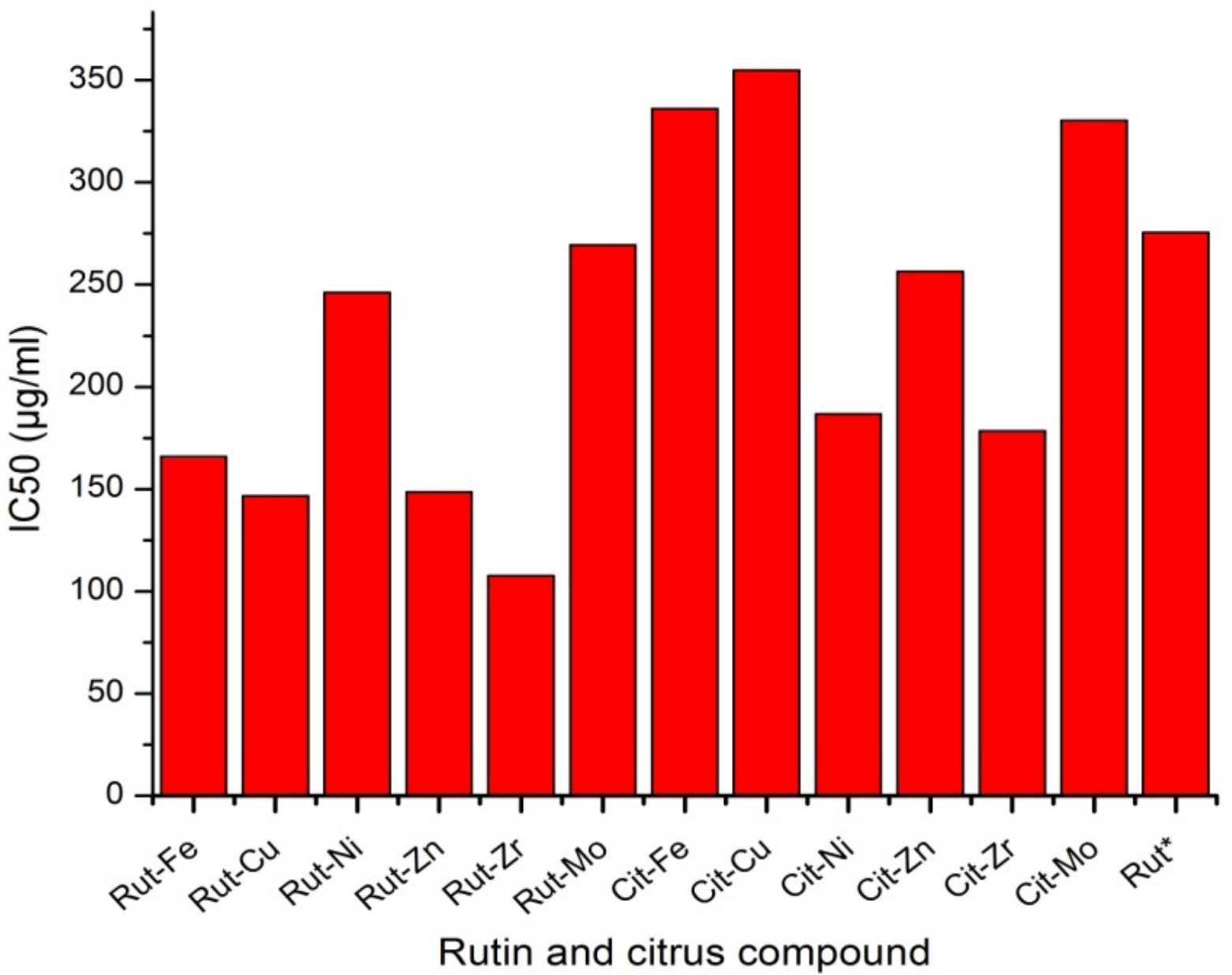

Figure 2H. IC50Metal complexes of Rutin and bioconjugate of citrus peel extract collected from Uttarakhand region for Metal chelating activity 


\section{Reducing power activity}

Mean percent reducing power activity of metal complexes of Rutin and bioconjugate of citrus peel extract in a dose depended manner with minimum at lower and maximum at higher dose level $(100 \mu \mathrm{g} / \mathrm{mL}-500 \mu \mathrm{g} / \mathrm{mL})$ are presented in Table9 and Fig 2 I. The radical scavenging potential of synthesized metal complexes in the form of their significant $(\mathrm{p}<0.05) \mathrm{IC}_{50}$ values revealed the order as Cit-Fe $>$ Rut-Cu $>$ Cit-Zr $>$ Cit-Ni $>$ Cit-Cu $>$ Cit- $Z n>$ Rut-Zr $>$ Cit-Mo $>$ Rut-Fe $>$ Rut-Mo $>$ Rut $-\mathrm{Zn}>$ Rut $-\mathrm{Ni}$ (Table 10Fig 2J) $\mathrm{IC}_{50}$ values of standards were found in the order as Rutin $\left(\mathrm{IC}_{50}=109.65\right)$.

Table 9. Percent reducing power activity of metal complexes of Rutin and Bioconjugate of citrus peel extract collected from Uttarakhand region at different concentrations

\begin{tabular}{|c|c|c|c|c|c|c|}
\hline \multirow{2}{*}{ S.No } & \multirow{2}{*}{$\begin{array}{c}\text { Metal } \\
\text { complexes }\end{array}$} & \multicolumn{5}{|c|}{$\%$ Reducingpower activity at $700 \mathrm{~nm}$} \\
\hline & & $100 \mu \mathrm{g} / \mathrm{mL}$ & $200 \mu \mathrm{g} / \mathrm{mL}$ & $300 \mu \mathrm{g} / \mathrm{mL}$ & $400 \mu \mathrm{g} / \mathrm{mL}$ & $500 \mu \mathrm{g} / \mathrm{mL}$ \\
\hline 1 & Rut-Fe & $45.97 \pm 0.32$ & $40.03 \pm 0.34$ & $32.10 \pm 0.12$ & $35.15 \pm 1.21$ & $25.52 \pm 0.13$ \\
\hline 2 & Rut-Cu & $68.13 \pm 0.11$ & $64.21 \pm 0.33$ & $52.05 \pm 0.77$ & $45.36 \pm 1.11$ & $23.44 \pm 0.11$ \\
\hline 3 & Rut-Ni & $66.02 \pm 0.40$ & $62.09 \pm 0.35$ & $50.03 \pm 0.09$ & $45.65 \pm 0.2$ & $25.43 \pm 0.34$ \\
\hline 4 & Rut-Zn & $68.23 \pm 0.51$ & $50.45 \pm 0.22$ & $30.02 \pm 0.08$ & $21.28 \pm 0.19$ & $18.20 \pm 3.04$ \\
\hline 5 & Rut-Zr & $65.21 \pm 0.81$ & $61.21 \pm 0.21$ & $51.54 \pm 0.03$ & $39.32 \pm 0.06$ & $25.20 \pm 1.20$ \\
\hline 6 & Rut-Mo & $67.02 \pm 0.22$ & $60.03 \pm 0.22$ & $45.32 \pm 0.04$ & $37.04 \pm 0.12$ & $25.40 \pm 0.06$ \\
\hline 7 & Cit-Fe & $58.06 \pm 0.03$ & $40.45 \pm 0.34$ & $36.26 \pm 0.07$ & $28.24 \pm 0.29$ & $23.40 \pm 1.23$ \\
\hline 8 & Cit-Cu & $62.03 \pm 0.07$ & $31.00 \pm 0.45$ & $25.20 \pm 0.23$ & $22.22 \pm 1.33$ & $20.04 \pm 2.11$ \\
\hline 9 & Cit-Ni & $53.1 \pm 0.06$ & $48.02 \pm 0.67$ & $43.12 \pm 0.01$ & $34.14 \pm 2.33$ & $20.10 \pm 0.67$ \\
\hline 10 & Cit-Zn & $43.1 \pm 0.44$ & $37.08 \pm 0.22$ & $31.09 \pm 0.01$ & $23.14 \pm 0.13$ & $18.07 \pm 0.99$ \\
\hline 11 & Cit-Zr & $58.1 \pm 0.11$ & $52.04 \pm 0.26$ & $45.06 \pm 0.03$ & $32.08 \pm 0.12$ & $20.09 \pm 0.34$ \\
\hline 12 & Cit-Mo & $45.34 \pm 0.12$ & $41.26 \pm 0.22$ & $30.28 \pm 0.04$ & $25.28 \pm 0.11$ & $20.0 \pm 0.22$ \\
\hline 13 & Rut* & $44.2 \pm 0.23$ & $40.20 \pm 0.11$ & $30.20 \pm 0.37$ & $25.24 \pm 2.65$ & $19.26 \pm 0.09$ \\
\hline
\end{tabular}

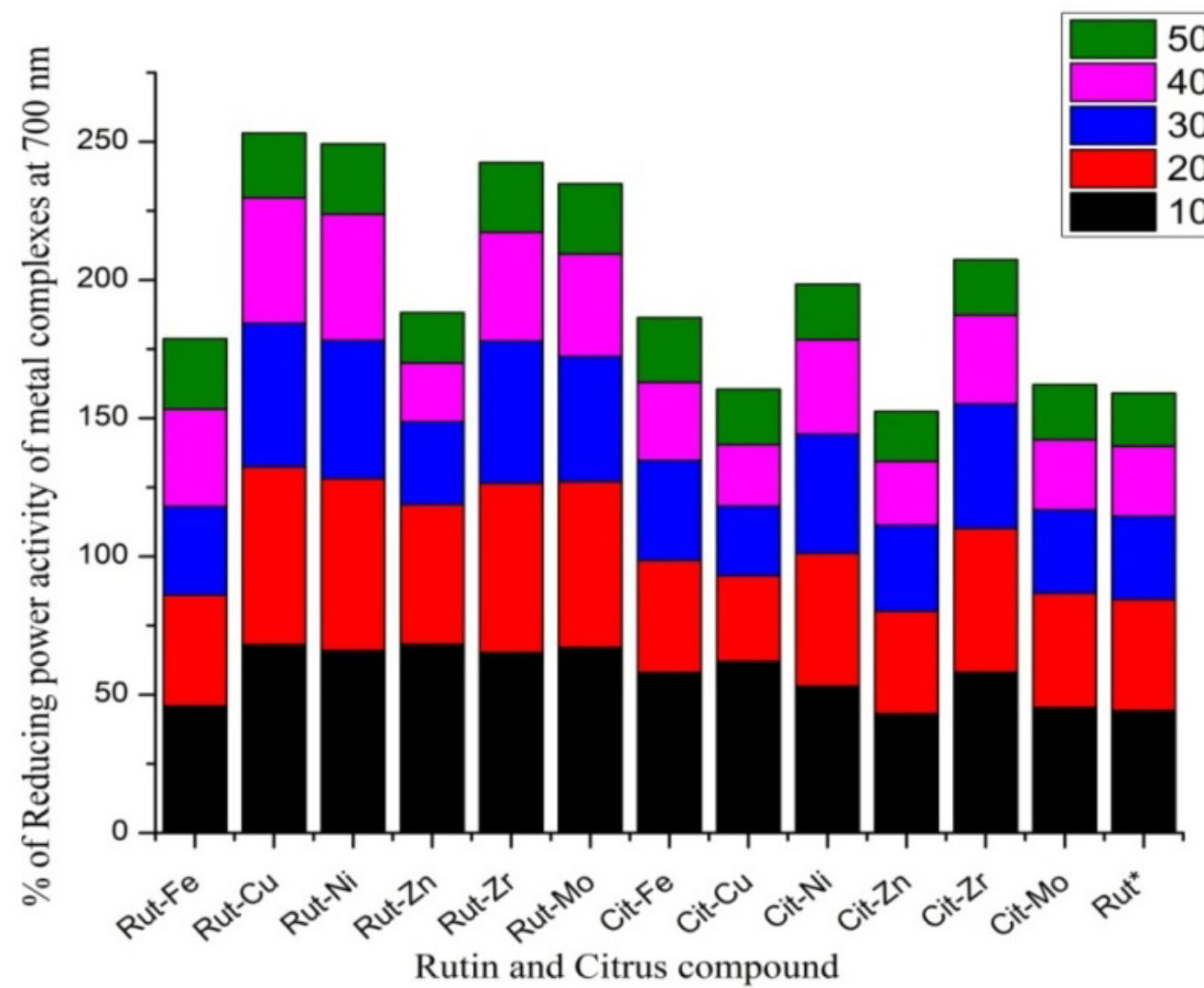

Figure 2I. Percent reducing power activity of metal complexes of Rutin and Bioconjugate of citrus peel extract collected from Uttarakhand region at different concentrations 
Table 10. $\mathrm{IC}_{50}$ of Metal complexes of Rutin and bioconjugate of citrus peel extract for reducing power activity collected from Uttarakhand region at different concentrations

\begin{tabular}{|c|c|c|c|c|c|}
\hline \multirow{2}{*}{ S.No } & \multirow{2}{*}{ Metal complexes } & \multicolumn{3}{|c|}{$\mathbf{R P}_{50}$ values $(\mu \mathrm{g} / \mathrm{mL})$ in triplicates } & \multirow{2}{*}{$\begin{array}{c}\text { Mean } \mathbf{R P}_{50} \text { values } \\
(\mu \mathrm{g} / \mathbf{m L})\end{array}$} \\
\hline & & $\mathbf{I}^{\text {st }}$ & II $^{\text {nd }}$ & III $^{\text {rd }}$ & \\
\hline 1 & Rut-Fe & 271.98 & 271 & 272.5 & $271.83 \pm 0.762^{\mathrm{d}}$ \\
\hline 2 & Rut-Cu & 63.27 & 63.27 & 62.08 & $62.87 \pm 0.687^{1}$ \\
\hline 3 & Rut-Ni & 306.48 & 306 & 305.5 & $305.99 \pm 0.49^{\mathrm{a}}$ \\
\hline 4 & Rut-Zn & 290 & 290 & 289.8 & $289.93 \pm 0.115^{b}$ \\
\hline 5 & Rut-Zr & 204.65 & 204 & 204.6 & $204.42 \pm 0.362^{\mathrm{f}}$ \\
\hline 6 & Rut-Mo & 287.72 & 287.72 & 287.5 & $287.65 \pm 0.127^{\mathrm{c}}$ \\
\hline 7 & Cit-Fe & 36.81 & 36.81 & 34 & $35.87 \pm 1.622^{\mathrm{m}}$ \\
\hline 8 & Cit-Cu & 144.93 & 144 & 145.5 & $144.81 \pm 0.757^{\mathrm{h}}$ \\
\hline ss9 & Cit-Ni & 107.82 & 107.82 & 105 & $106.88 \pm 1.628^{j}$ \\
\hline 10 & Cit-Zn & 172.91 & 172.95 & 175.6 & $173.82 \pm 1.542^{\mathrm{g}}$ \\
\hline 11 & Cit-Zr & 91.54 & 92.5 & 92.8 & $92.28 \pm 0.658^{\mathrm{k}}$ \\
\hline 12 & Cit-Mo & 211.25 & 210.92 & 210 & $210.72 \pm 0.648^{\mathrm{e}}$ \\
\hline 13 & Rut* & 110.35 & 109.2 & 109.4 & $109.650 .614^{\mathrm{i}}$ \\
\hline
\end{tabular}

Values are mean of three replicates \pm Standard deviation. Within column, mean values followed by the same letter are not significantly different according to Tukey's test $(\mathrm{p}<0.05)$.

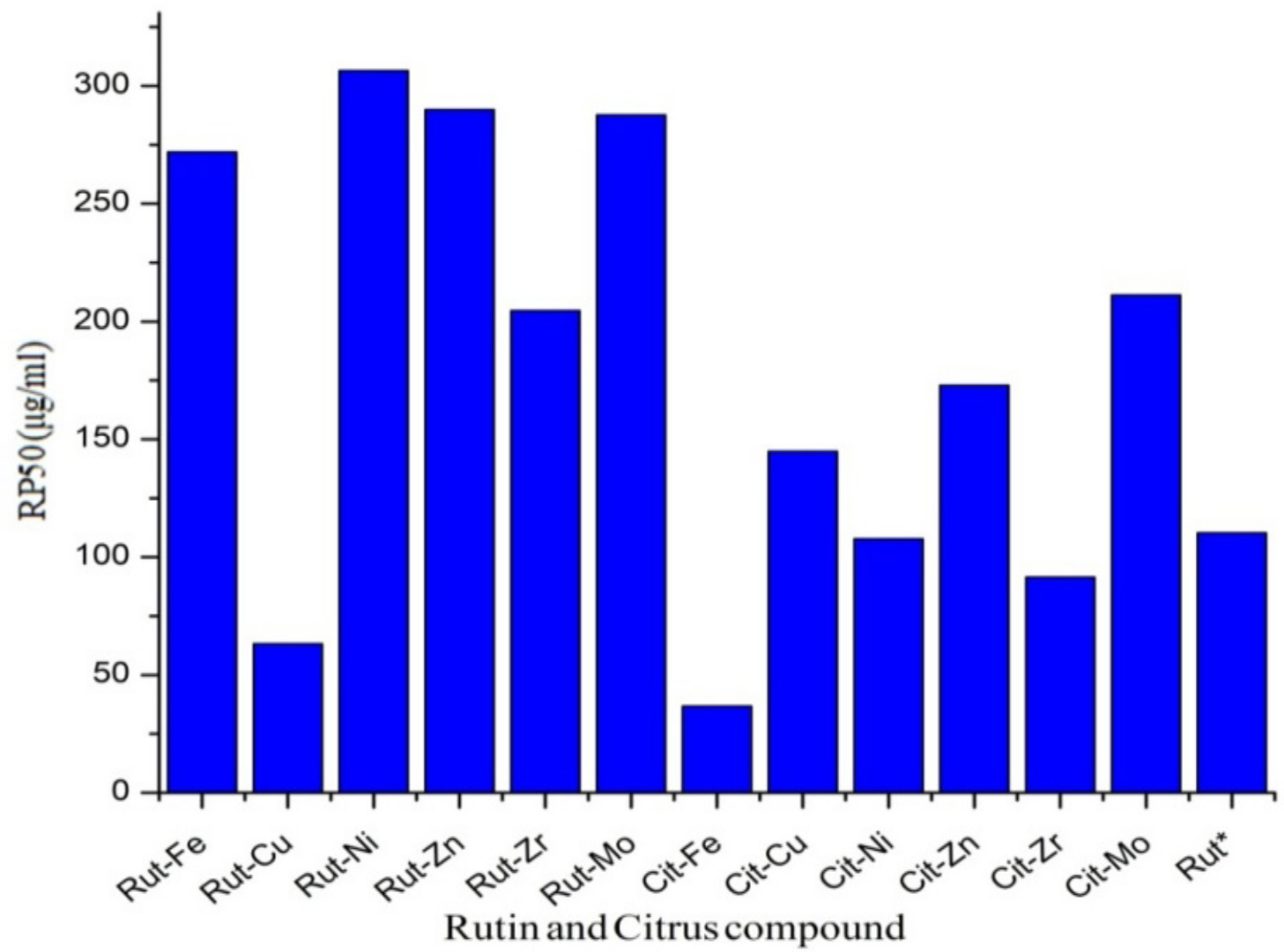

Figure 2J. $\mathrm{IC}_{50}$ of Metal complexes of Rutin and bioconjugate of citrus peel extract collected from Uttarakhand region for reducing power activity 
Synthesis and characterization of metal complexes of Rutin and bioconjugate of citrus peel extract

Table 11. UV-visible Spectroscopy Analysis of metal complexes of Rutin

\begin{tabular}{|c|c|c|c|}
\hline S.No & Complexes & I -Band (nm) & II -Band (nm) \\
\hline 1 & Rutin & 300 & 500 \\
\hline 2 & Rut-Fe & 220 & 430 \\
\hline 3 & Rut-Cu & 250 & 400 \\
\hline 4 & Rut-Mo & 240 & 410 \\
\hline 5 & Rut-Zr & 260 & 420 \\
\hline 6 & Rut-Ni & 220 & 410 \\
\hline 7 & Rut-Zn & 300 & 480 \\
\hline
\end{tabular}
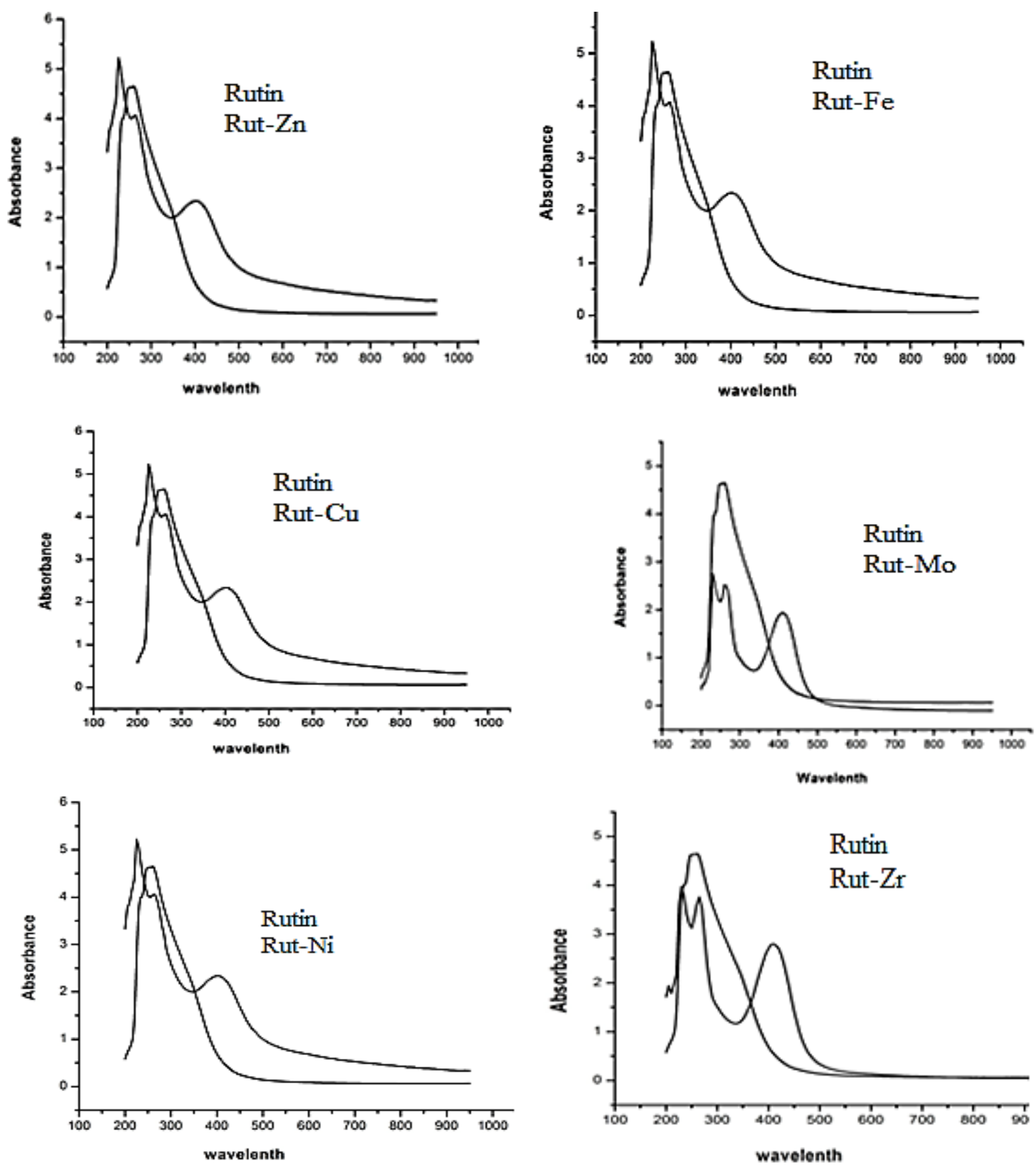

Figure 3A. UV- visible spectra of metal complexes of Rutin 
Table 12. UV-visible Spectroscopy Analysis of bioconjugate of citrus extract

\begin{tabular}{|c|c|c|c|}
\hline S.No & Complexes & I -Band (nm) & II -Band (nm) \\
\hline 1 & Citrus & 340 & 420 \\
\hline 2 & Citrus -Fe & $300-350$ & 450 \\
\hline 3 & Citrus -Cu & $200-240$ & 400 \\
\hline 4 & Citrus -Mo & $210-240$ & 440 \\
\hline 5 & Citrus -Zr & 300 & 350 \\
\hline 6 & Citrus -Ni & $300-340$ & 480 \\
\hline 7 & Citrus-Zn & $240-280$ & 450 \\
\hline
\end{tabular}
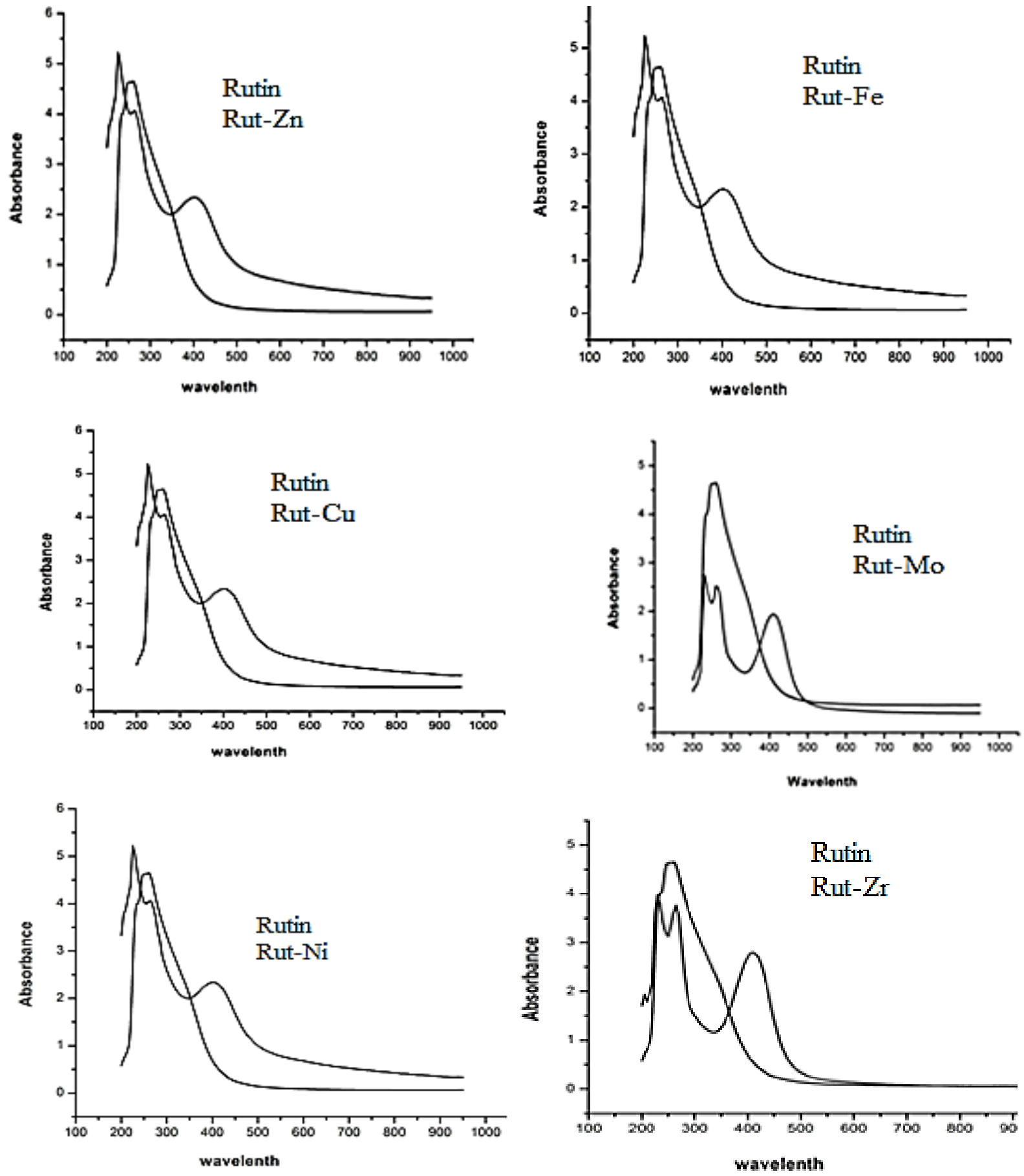

Figure 3B. UV- visible spectra of metal complexes of Rutin 
Rutin has ability to effectively chelate the metal ions via o-dyhydroxyl and 5OH-4CO groups, but involvement of particular site in chelate formation depends more on the nature of a metal ion and some other factors such as location of hydroxyl groups and steric hindrance at legating groups to affect the complexation it has been noticed that the changes in the visible spectrum of rutin $(350-500 \mathrm{~nm})$ depend more on the nature of metal salt, complex present and other factors. In general, the spectral changes in the 250-270 $\mathrm{nm}$ range are insignificant whereas, the changes in the visible range $(350-500 \mathrm{~nm})$ are highly significant for complexation purpose (N. B. Mel'nikova et al., 2002). Similar to that, electronic spectra of rutin and its complexes show two clear visible peaks. The absorbance peaks in the visible region at 359, 422 and $413 \mathrm{~nm}$ are produced whilst those visualized at lower region. Around 257, 275 and 271 $\mathrm{nm}$ is associated to a ring (i.e. benzoyl system) for rutin and its UV-Vis absorption spectra of ligand Rutin and their corresponding different metal complexes $(\mathrm{Fe}, \mathrm{Cu}, \mathrm{Mo}, \mathrm{Ni}$, $\mathrm{Zr}$ and $\mathrm{Zn}$ ) of Rutin were recorded in the range of 300 to $500 \mathrm{~nm}$ in DMSO (Table 11).

The shifting of bands between the ligand and metal complexes of ligand and the transition metals. Appearance of new absorption bands gives an indication of the formation of the complexes. The UV-visible spectrum of Rutin showed an instance absorbance band at $300 \mathrm{~nm}$ and transition between 300 to $500 \mathrm{~nm}$ found to be maximum at $320 \mathrm{~nm}$. The UV-visible spectrum of the zinc complex shows a broad absorption band at $420 \mathrm{~nm}$ attributed to the bathochromic shift and the lower wavelength exhibited to the hypsochromic shift. The Iron complex shows a broad absorption band at $400 \mathrm{~nm}$ attributed to the bathochromic shift and the lower wavelength $200 \mathrm{~nm}$ exhibited to the hypsochromic shift. In Copper complex the hypsochromic shift $250 \mathrm{~nm}$ while absorption at 380 to $420 \mathrm{~nm}$ exhibited shoulder like bathochromic shift. In the UV spectrum of
Mo and Zr The spectra show the bathochromic shift of about 410 and $430 \mathrm{~nm}$ respectively for $\mathrm{Mo}^{+6}, \mathrm{Zr}^{4+}$ and Such spectral shifts are either analogous to $\pi-\pi^{*}$ electronic transitions or witness the presence of charge transfer complexes (CTC) produced through the formation of coordination bonds between unshared electron pairs of oxygen atoms of the phenolic groups at rutin and the d-orbital's of molybdenum and zirconium ions.

UV-Vis absorption spectra of bioconjugate of citrus peel extract were recorded in the range of 300 to $550 \mathrm{~nm}$ in DMSO corresponding to metal ( $\mathrm{Fe}, \mathrm{Cu}, \mathrm{Mo}, \mathrm{Ni}, \mathrm{Zr}$ and $\mathrm{Zn}$ ) in Table 12. The shifting of bands between the ligand and metal complexes of ligand and the transition metals. Appearance of new absorption bands gives indications of the formation of the complexes. The UV-visible spectrum of citrus peel extract showed broad absorbance band at 310 $\mathrm{nm}$ and transition between 300 to $500 \mathrm{~nm}$ found to be maximum at $320 \mathrm{~nm}$. The UV-visible spectrum of the zinc complex shows a broad absorption band at $420 \mathrm{~nm}$ attributed to the bathochromic shift and the lower wavelength exhibited to the hypsochromic shift. The Iron complex shows a broad absorption band at $380 \mathrm{~nm}$ attributed to the bathochromic shift and the lower wavelength $300 \mathrm{~nm}$ exhibited to the hypsochromic shift. In Copper complex the hypsochromic shift $250 \mathrm{~nm}$ while absorption at 380 to $420 \mathrm{~nm}$ exhibited shoulder like bathochromic shift. In the UV spectrum of Mo and Zr The spectra show the bathochromic shift of about 400 and 320 $\mathrm{nm}$ respectively for $\mathrm{Mo}^{+6}, \mathrm{Zr}^{4+}$ and such spectral shifts are either analogous to $\pi-\pi^{*}$ electronic transitions or witness the presence of charge transfer complexes (CTC) produced through the formation of coordination bonds between unshared electron pairs of oxygen atoms of the phenolic groups at rutin and the d-orbital's of molybdenum and zirconium ions. 

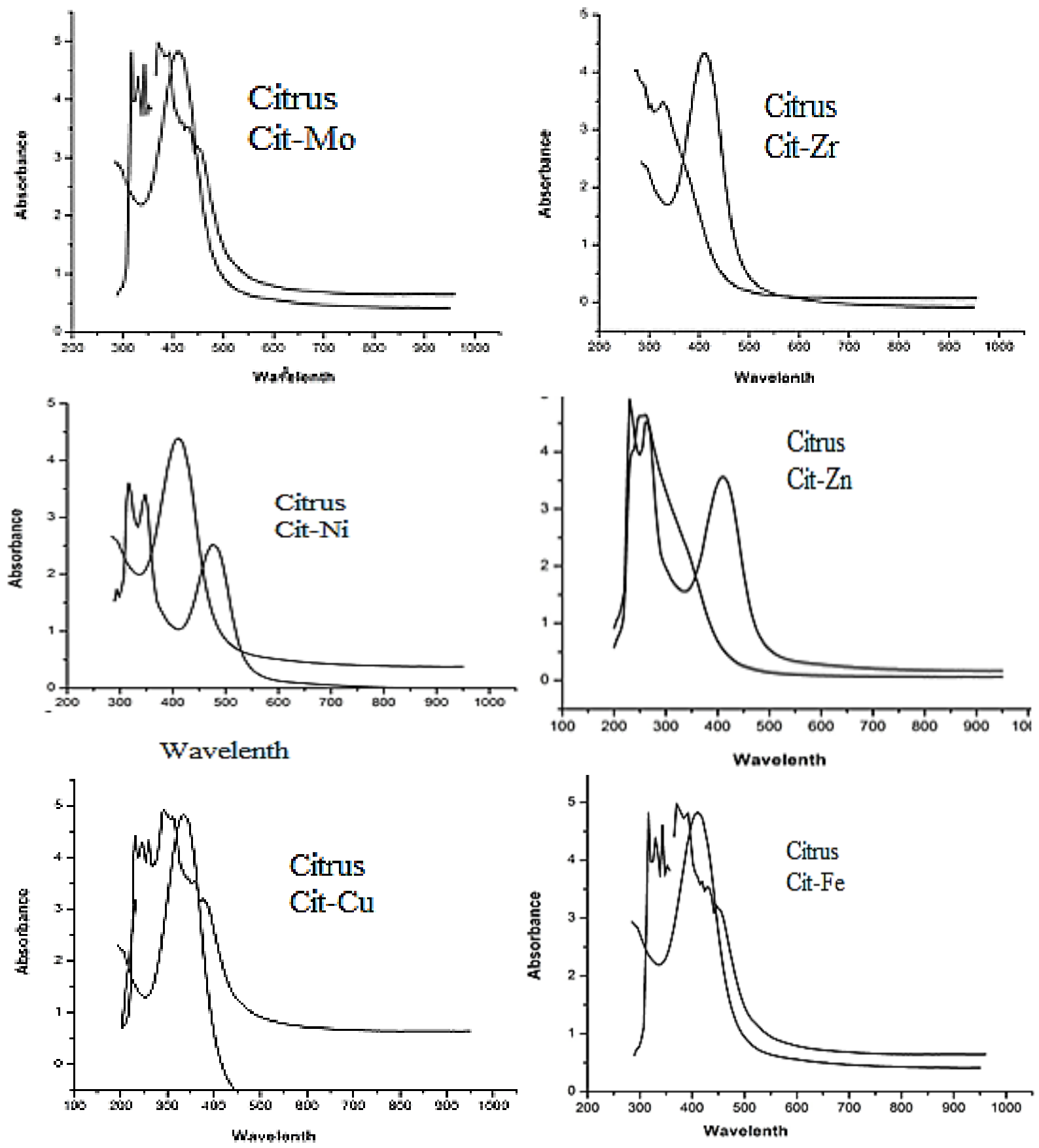

Figure 3C. UV-visible spectra of metal complexes of bioconjugate of citrus peel extract

\section{Optical properties of metal complexes}

During absorption process, transition of electron takes place from a lower to higher energy state by absorbing a photon of definite energy, depends upon the transmitted radiation. The fundamental absorption shows a sudden rise in the absorption, known as absorption edge, which can be used to determine the optical band gap (Eg) is calculated from the relation (Tauc,s plot) from the following equation:

$$
\mathrm{ah \vartheta}=\mathrm{A}(\mathrm{h} \vartheta-\mathrm{Eg})^{\mathrm{n}}
$$

Where $\mathbf{n}$ is equal to $1 / 2$ and 2 for direct and indirect transition respectively. 


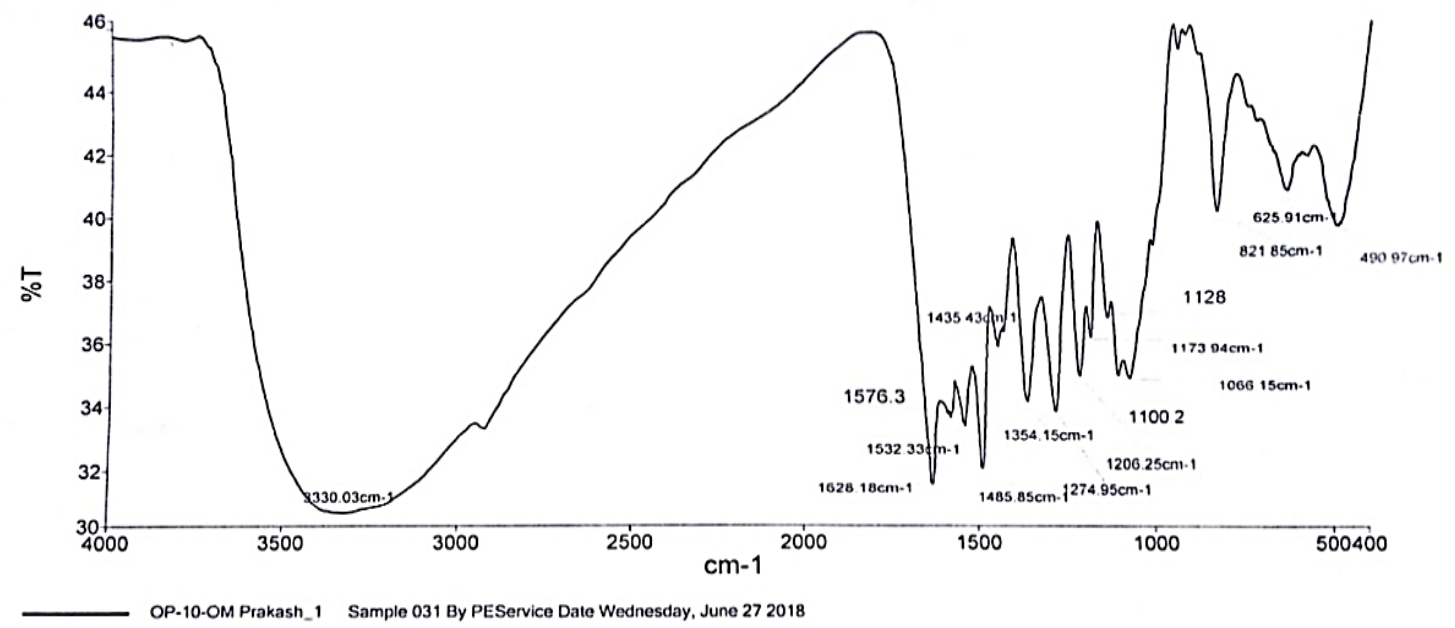

Figure 4A. FT-IR bioconjugate of Cit-Fe

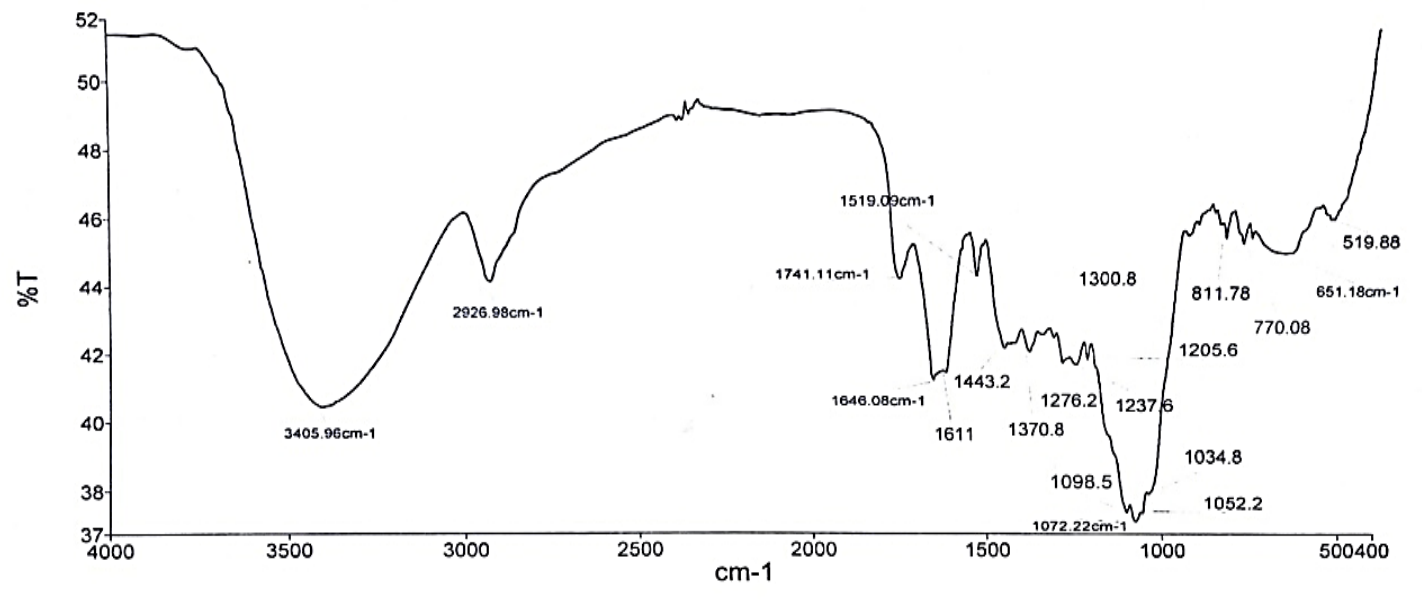

OP-12-OM Prakash_1 Sample 073 By PEService Date Wednesday. June 272018

Figure 4B. FT-IR of bioconjugate Cit-Mo

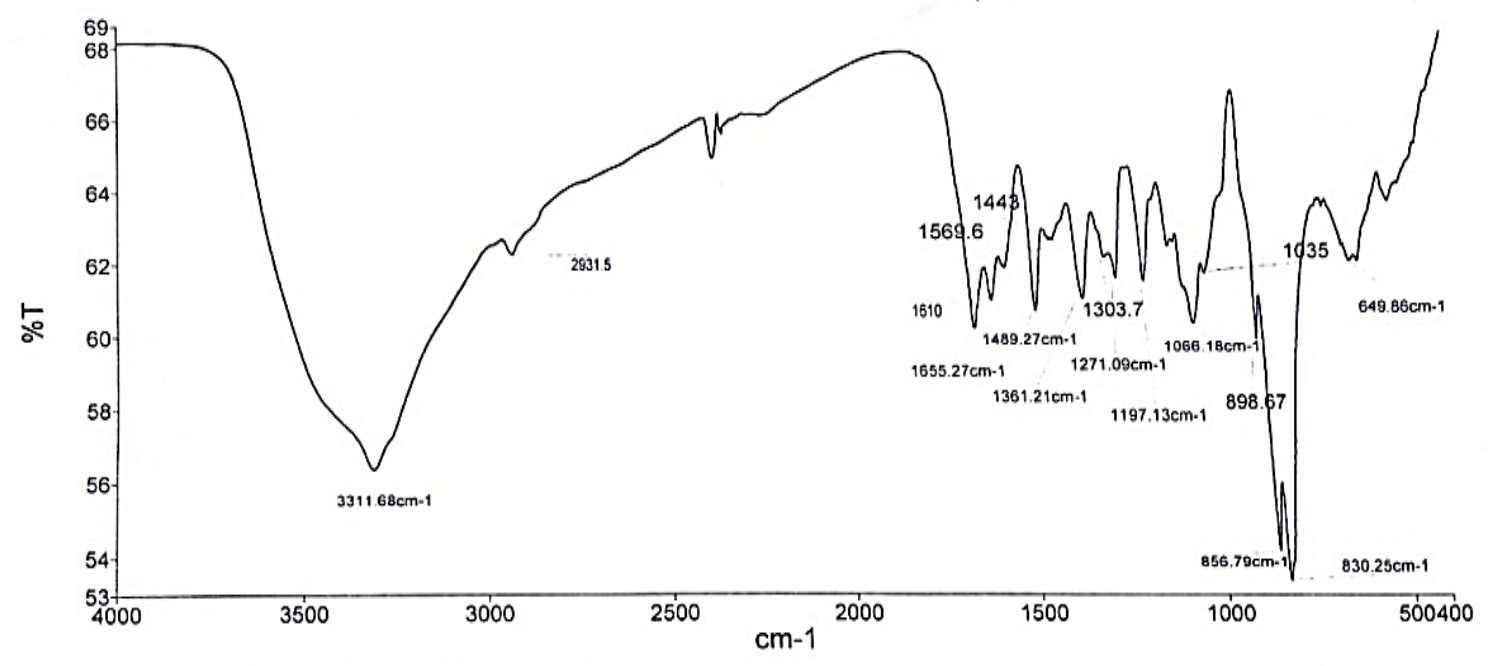

OP-13-OM Prakash_1 Sample 075 By PEService Date Wednesday, June 272018

Figure 4C. FT-IR of bioconjugate of Cit-Ni 


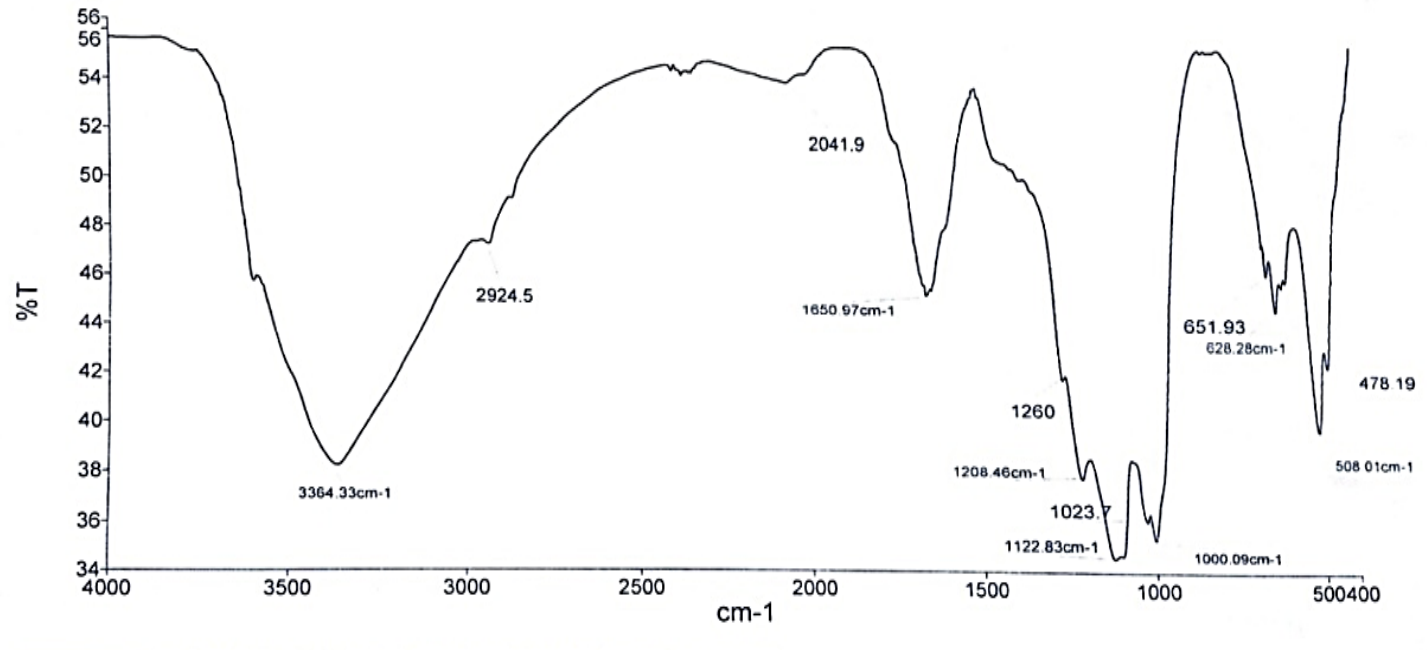

OP-4-OM Prakash_1 Sample 072 By PEService Date Monday, June 252018

Figure 4D. FT-IR of Metal complex of Rut-Zn

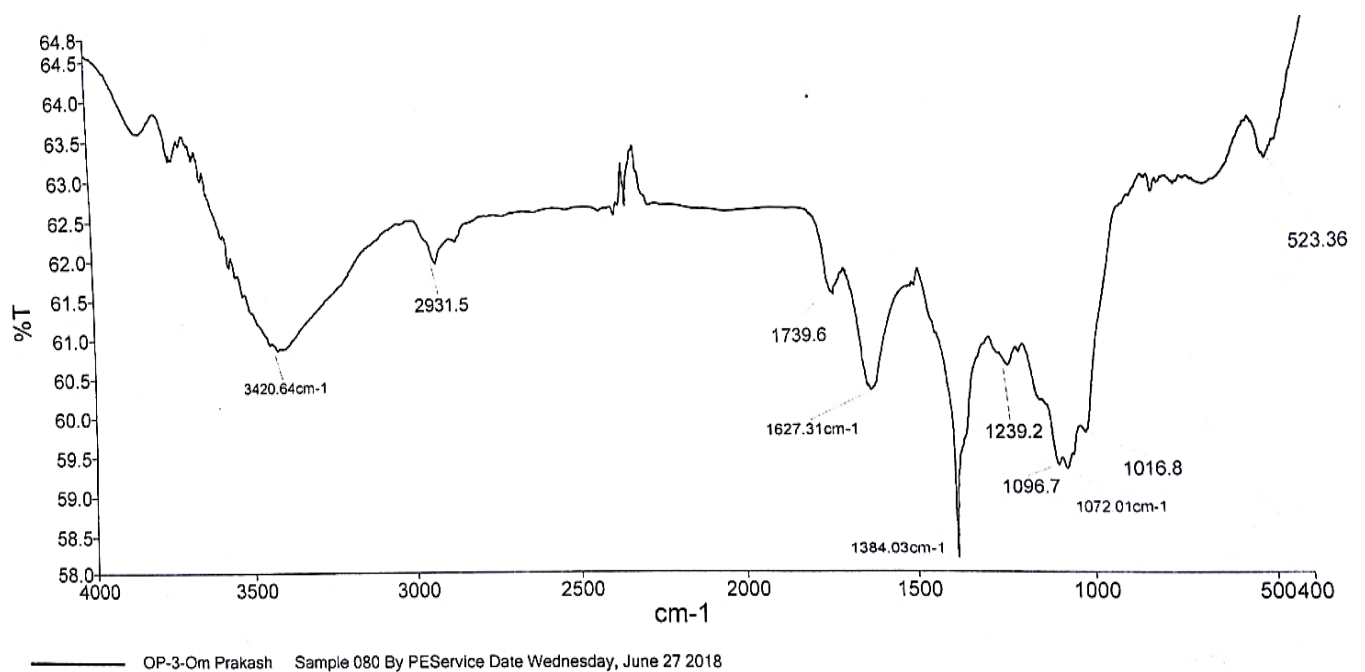

Figure 4E. FT-IR of metal complex Rut-Ni

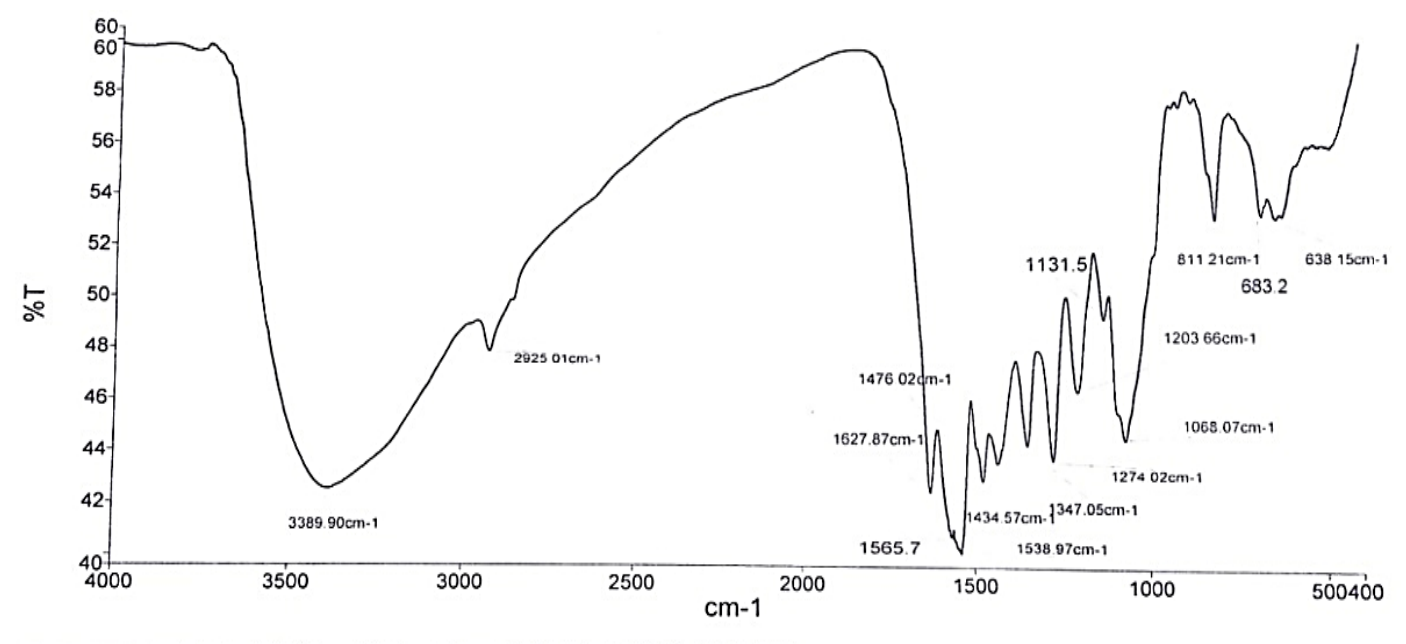

OP-9-OM Prakash_1 Sample 028 By PEService Date Wednesday, June 272018

Figure 4F. FT-IR of bioconjugate of Cit-Zn 


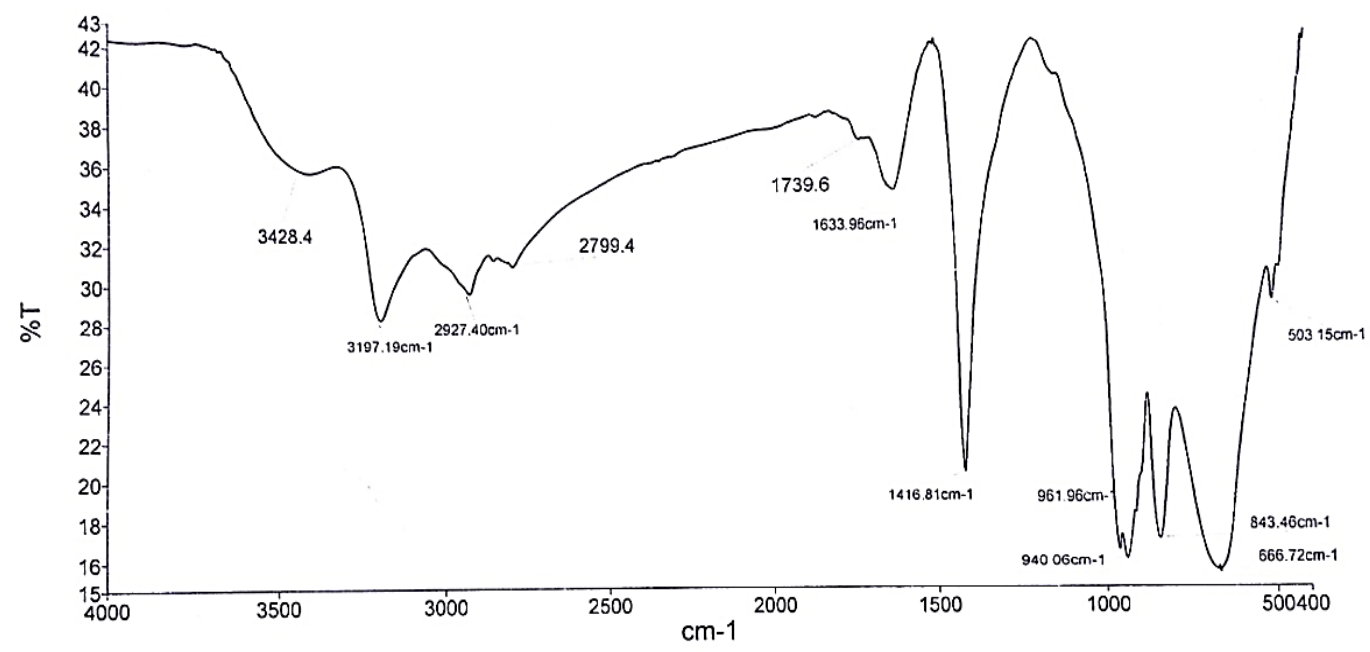

OP-1-OM Prakash_1 Sample 004 By PEService Date Monday, June 252018

Figure 4G. FT-IR of metal complex Rut-Cu

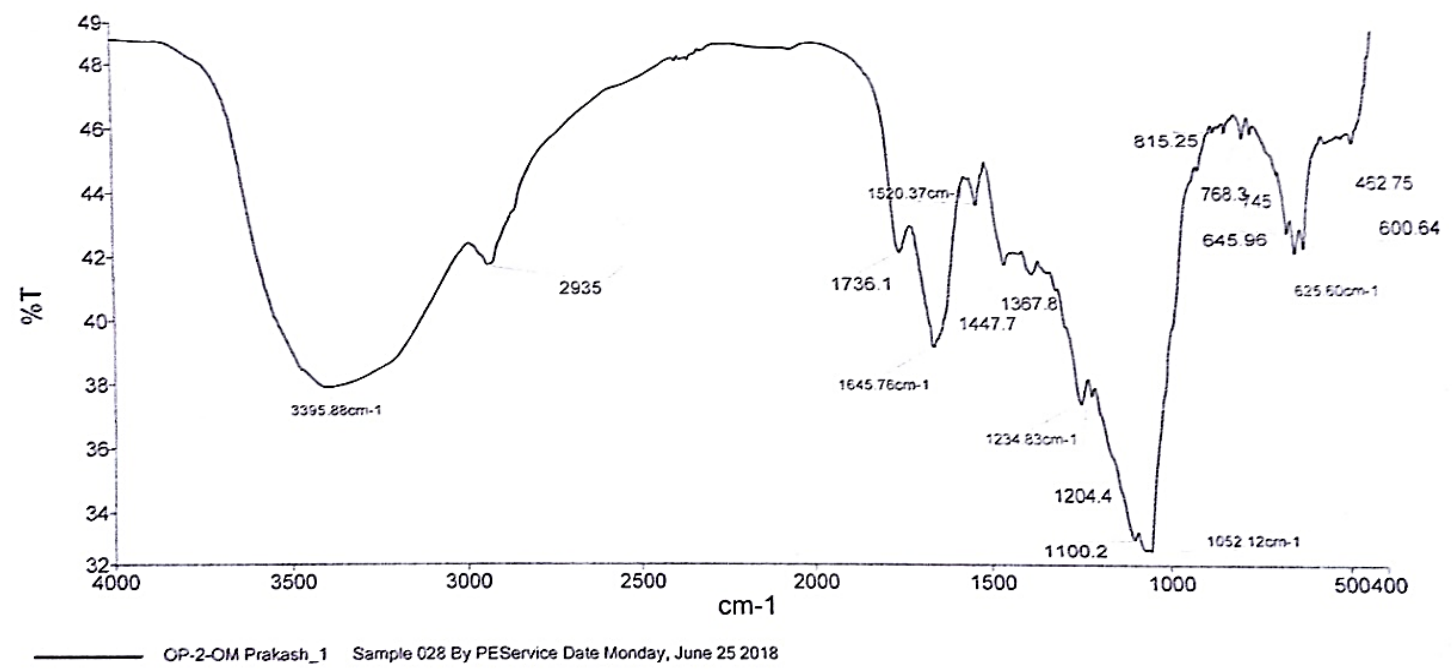

Figure 4H. FT-IR of metal complex Rut-Fe

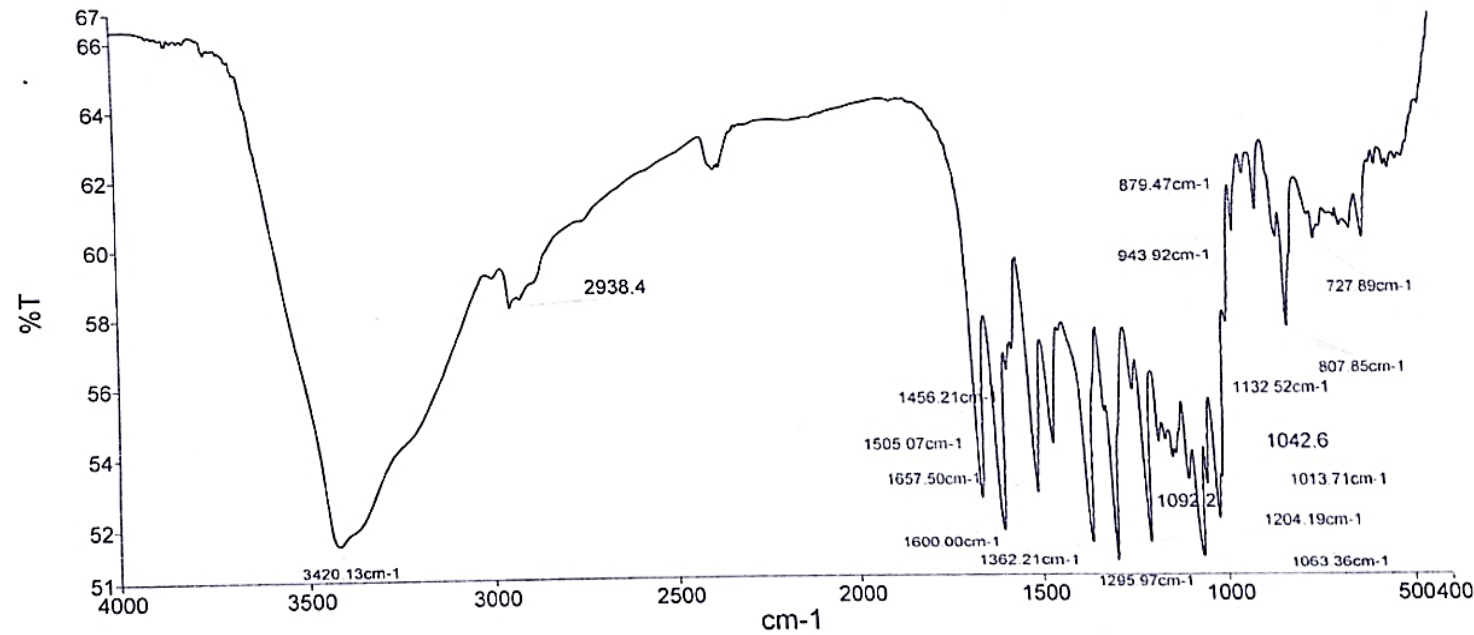

OP-11-OM Prakash_1 Sample 072 By PEService Date Wednesday, June 272018

Figure 4I. FT-IR metal complex of Cit-Cu 


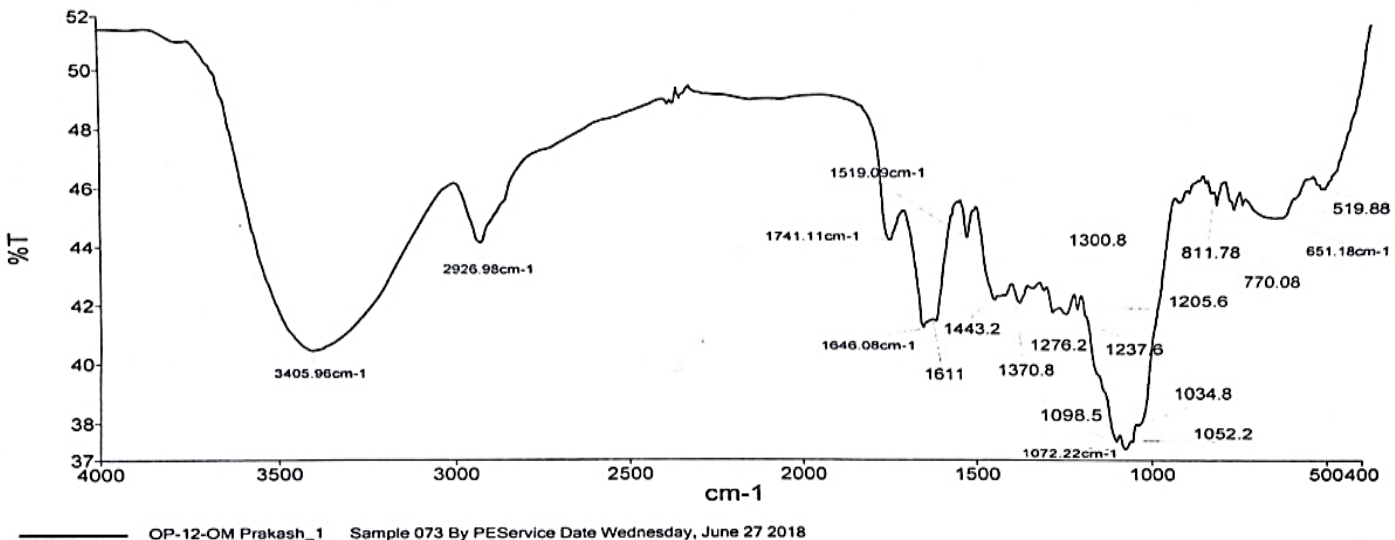

Figure 4J. FT-IR of metal complex Rut-Mo

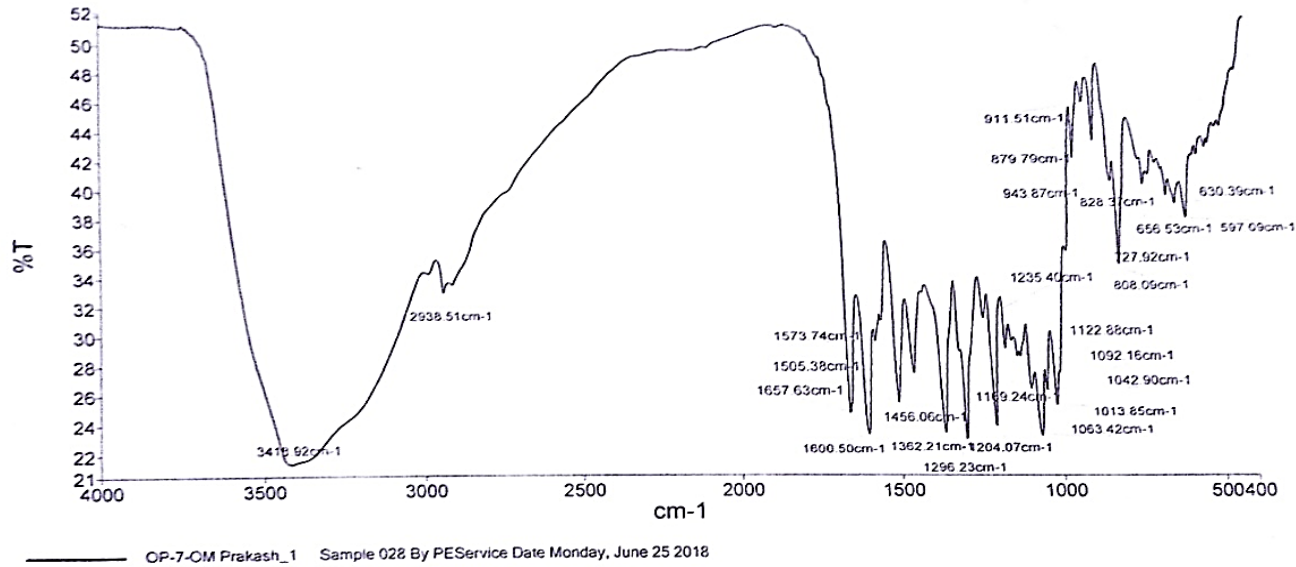

Figure 4K. FT-IR of Rutin

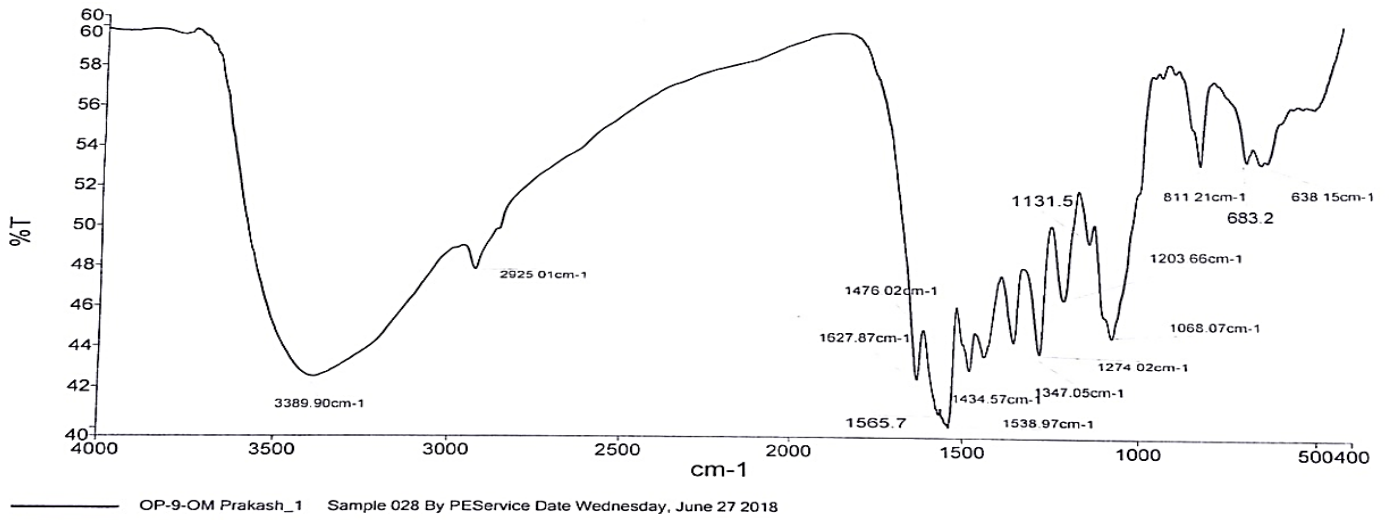

Figure 4L. FT-IR of Citrus

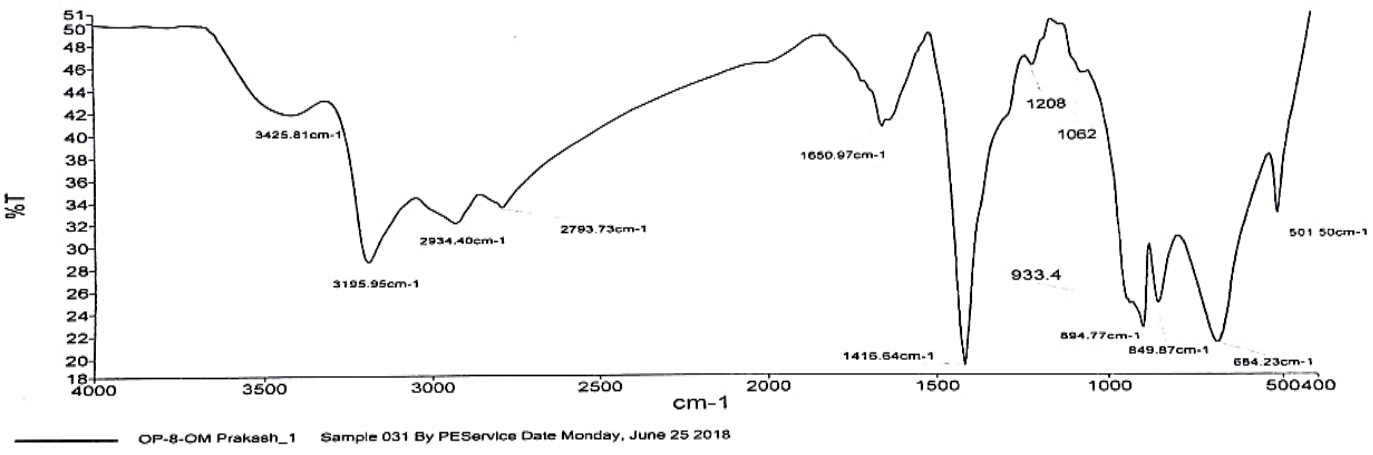

Figure 4M. FT-IR of metal complex of Rut-Zr 


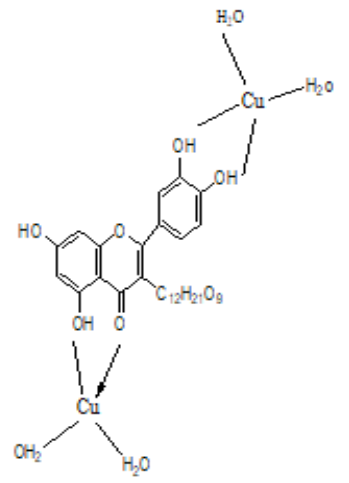<smiles>[M]CCOc1c(-c2ccc(OC)c(OC)c2)oc2cc(O)cc(O)c2c1=O</smiles>

Rutin

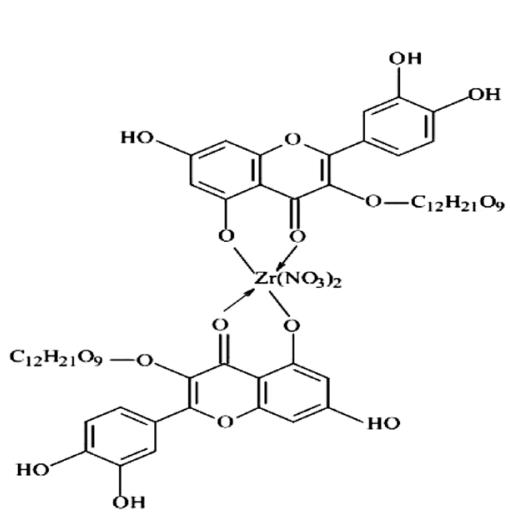

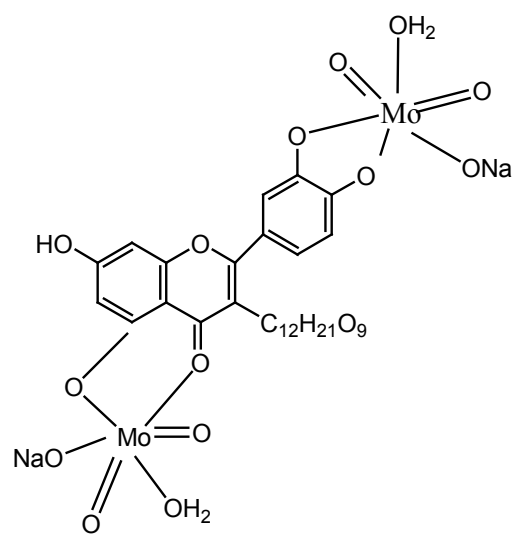

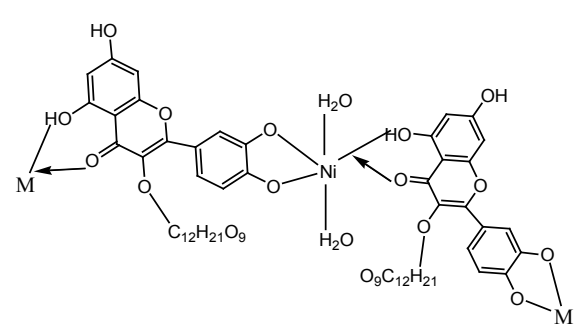

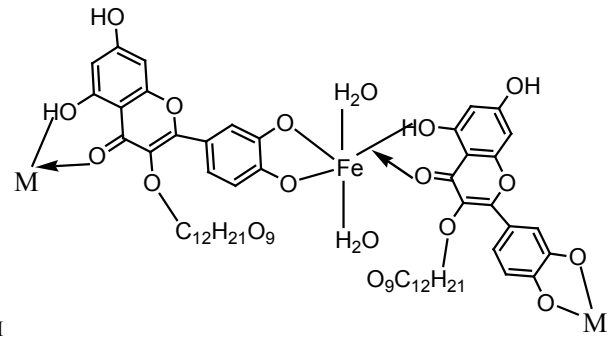

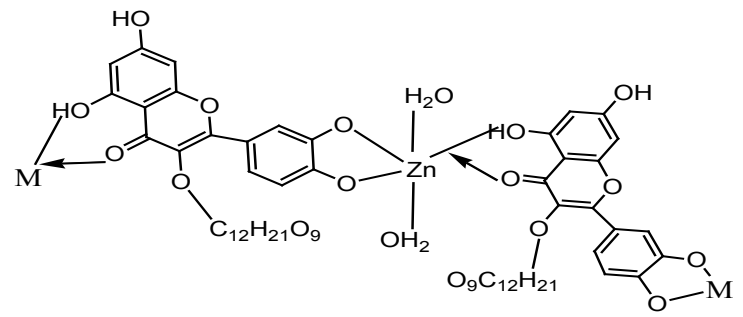

Figure 5. Metal complexes of Rutin with (Cu, Fe, Zn, Ni, Mo and $\mathrm{Zr}$ )

To systematically study the effects of the number of $d$ electrons of the first transition metal ions $(\mathrm{Fe}, \mathrm{Co}, \mathrm{Ni}, \mathrm{Cu}$, $\mathrm{Zr}$, Mo and $\mathrm{Zn}$ ) on the formation and stability of metal flavonoid complexes of Rutin $/ \mathrm{M}^{2+}, \mathrm{M}^{4+}, \mathrm{M}^{6+}$ complex as a model system to investigate the structures and properties of these complexes. The FT-IR spectrum(Fig 4A-4M) of ligand Rutin shows characteristic absorption bands at 3410 $\mathrm{cm}^{-1}$ corresponding to hydroxyl stretching
$\mathrm{v}(\mathrm{O}-\mathrm{H}),(\mathrm{C}-\mathrm{H}) \mathrm{sp}^{3} \quad$ stretching, $\quad \mathrm{v}(\mathrm{C}-\mathrm{H}) \mathrm{sp}^{2} 2938 \quad \mathrm{~cm}^{-1}$ stretching, carbonyl stretching $3418 \mathrm{~cm}^{-1}, \mathrm{v}(\mathrm{C}=\mathrm{C})$ aromatic ring stretching $1610 \mathrm{~cm}^{-1}, \mathrm{v}(\mathrm{C}-\mathrm{C}) 1100 \mathrm{~cm}^{-1}$ stretching, $\mathrm{v}(\mathrm{C}-\mathrm{O}) 1300 \mathrm{~cm}^{-1}$ phenolics, $\mathrm{v}\left(\mathrm{OCH}_{3}\right) 1130 \mathrm{~cm}^{-1}$ stretching respectively. Band at $3410 \mathrm{~cm}^{-1}$ of phenolic $\mathrm{v}(\mathrm{O}-\mathrm{H})$ stretching shiftedbroad peak in Nickel, Iron, Copper, and Molybdenum complexes with a large shifting in case of Zirconiumand Zinc complexes. The different modes of 
vibration of $\mathrm{C}-\mathrm{C}, \mathrm{C}=\mathrm{C}$ and $\mathrm{C}-\mathrm{H}$ affected the complex, probably due to the aromaticity of the formed chelate is different from the ligand Malešev and Kuntić (2007). In case of copper complex, the $\mathrm{v}(\mathrm{C}=\mathrm{O})$ carbonyl stretching is shifted to about $40 \mathrm{~cm}^{-1}$ of small peak which ensure the binding of copper metal. In case of iron complexes, the $\mathrm{v}(\mathrm{C}=\mathrm{O})$ band shifted to sharp peak of lower frequency at $1000 \mathrm{~cm}^{-1}, \mathrm{v}\left(\mathrm{OCH}_{3}\right)$ stretching band shifts to $1000 \mathrm{~cm}^{-1}$ and slight shifting of $\mathrm{v}(\mathrm{O}-\mathrm{H})$ band confirms the binding of $\mathrm{Fe}$ metal with these groups. But in case of zinc, zirconium and molybdenum complexes there is complete disappearance of $\mathrm{v}(\mathrm{C}=\mathrm{O})$ band, resulted that metals copper, zinc, and iron binds with oxygen of carbonyl groups. Such data favours that the carbonyl group $\mathrm{v}(\mathrm{C}=\mathrm{O})$ is strongly affected on coordination with transition (Yaul et al., 2009). In nickel complex the band of phenolic $\mathrm{v}(\mathrm{O}-\mathrm{H})$ and $\mathrm{v}\left(\mathrm{OCH}_{3}\right)$ stretching found to be shifted which confirms the binding of Ni metal with these groups. These bands are affected on coordination with metal ions, to point that the oxygen atom is a centre for coordination with the metal ions.

Low frequency IR spectra of the metal complexes provide very useful information regarding the type of metal-ligand bond arising from the coordination of ligands to the metal centre. On the other hand, the infrared spectra exhibit weak bands at the range $\left(1050\right.$ to $\left.1340 \mathrm{~cm}^{-1}\right)$, which could be assigned to the stretching frequencies of the $\mathrm{v}(\mathrm{M}-\mathrm{O})$. As shown in Fig the characteristic absorption bands of $\mathrm{Zn}-\mathrm{Cu}$ observed at $\mathrm{cm}^{-1}$ which is quite similar to earlier findings. Likewise for zinc complexes the metal to oxygen peak appeared at $\mathrm{cm}^{-1}$ supported by earlier studies (Panhwar and Memon, 2014).FT-IR peak at $\mathrm{cm}^{-1}$ confirming bonding between copper metal and oxygen. FT-IR of the nickel complex showed bands in $\mathrm{cm}^{-1}$ regions that may be attributed to (Ni-O) stretching, new bands in the Zinc complex at $540 \mathrm{~cm}^{-1}$ may indicate the formation of metal oxygen (M-O) bond Infrared absorption frequencies of citrus methanolic extract and bioconjugate of citrus extract. On comparing the spectra of citrus extract with their respective complexes, important distinctions are revealed. The FT-IRspectrum of citrus methanolic extract shows characteristic absorption peaks at $3670 \mathrm{~cm}^{-1}$ corresponding to phenolic $\mathrm{v}(\mathrm{O}-\mathrm{H}), \mathrm{v}(\mathrm{C}-\mathrm{H}) \mathrm{sp}^{3} 3000$ $\mathrm{cm}^{-1}$ stretching, $\quad \mathrm{v}(\mathrm{C}-\mathrm{H}) \quad \mathrm{sp}^{2} 3100 \quad \mathrm{~cm}^{-1}$ stretching of flavonoids/phenolics, $\quad \mathrm{v}(\mathrm{C}=\mathrm{O}) \quad 1720 \quad \mathrm{~cm}^{-1}$ carbonyl stretching of aldehydes or ketenes, $\mathrm{v}(\mathrm{C}=\mathrm{C}) \quad 1600$ $\mathrm{cm}^{-1}$ aromatic ring stretching, $\mathrm{v}(\mathrm{OH}) 3600 \mathrm{~cm}^{-1}$ alcoholic stretching and $\mathrm{v}(\mathrm{R}-\mathrm{O}-\mathrm{R}) \quad 1000 \quad-1300 \quad \mathrm{~cm}^{-1}$ ether respectively.

A broad band appears in the spectra of the citrus methanolic extract at $87 \mathrm{~cm}^{-1}$ which shifted between $\mathrm{cm}^{-1}$ in all metal complexes. In copper, zinc, cobalt and iron complexes this band shifted to lower wave number with zinc complex appearing at $3800 \mathrm{~cm}^{-1}$ while in case of iron and nickel complexes it shifted to higher frequency. A strong band at about $3566.4 \mathrm{~cm}^{-1}$ detected in the spectra of the citrus methanolic extract assigned to $\mathrm{v}(\mathrm{C}=\mathrm{O})$ stretching shifted in the spectra of metal complexes indicates that the coordination occurs through the $\mathrm{C}=\mathrm{O}$ oxygen atom. Complexes of copper, zinc, nickel, and copper causes red shift in $\mathrm{v}(\mathrm{C}=\mathrm{O})$.

Metal-ligand vibrations are mostly observed below $800 \mathrm{~cm}^{-1}$ (Roy, S et al., 2015). FT-IR peaks observed at lower wave number between 450 to $650 \mathrm{~cm}^{-1}$ in all complexes, which is not measured in the free ligand spectrum.

\section{Conclusions}

Plants are the rich source of drugs used in primary health-care. The main advantages of using plants as drugs is safe, low cost and more reliable than the synthetic products; Hence plants can be used as effective pharmacological agents, there is a growing demand for food that is free of synthetic chemicals as preservatives, it is necessary to examine and identify alternatives and safe approaches for controlling food born pathogen. The use of natural compounds from plants could open up the possibility of using them as novel antimicrobials in food system. The results of the present study support the recycling of fruit waste. Thereby, yielding new products and meeting the requirements of essential products required in human, animal and plant nutrition as well as in the pharmaceutical industry.

\section{Acknowledgement}

Thankful to the G.B. Pant University of Agriculture and Technology, Pantnagar (India) for providing the necessary facilities and assistance required for completion of this work.

\section{REFERENCES}

[1] Prakash, Om., Dhanik, Jyotsna., Belal, Babita., Verma, Anil., Joshi, Hem. C. and Vivekanand. 2018. Antimicrobial activity of different Citrus species against different pathogenic bacteria. International Journal of Chemical Studies. 6(3): 3023-3029.

[2] Barku, V. Y. A., Opoku-Boahen, Y., Owusu-Ansah, E. and Mensah, E. F.2013. Antioxidant activity and the estimation of total phenolic and flavonoid contents of the root extract of Amaranthus spinosus. Asian Journal of Plant Science and Research, 3(1): 69-74.

[3] Proestos, C., Boziaris, I. S., Nychas, G. J. and Komaitis, M. 2006. Analysis of flavonoids and phenolic acids in Greek aromatic plants: Investigation of their antioxidant capacity and antimicrobial activity. Food Chemistry, 95(4):664-671.

[4] Sharma, S., Ali, A., Ali, J., Sahni, J. K. and Baboota, S. 2013. Rutin: therapeutic potential and recent advances in drug 
delivery. Expert Opinion on Investigational Drugs.22(8):1063-1079.

[5] Pan, S. Y., Litscher, G., Gao, S. H., Zhou, S. F., Yu, Z. L., Chen, H. Q. and Ko, K. M. 2014. Historical perspective of traditional indigenous medical practices: the current renaissance and conservation of herbal resources. Evidence-Based Complementary and Alternative Medicine. 4(3): 172-179.

[6] Wang, K. T. 2015. Research design in counseling.Nelson Education. pp. 15-21.

[7] Kreft, S., Knapp, M. and Kreft, I. 1999.Extraction of rutin from buckwheat (Fagopyrumesculentum Moench) seeds and determination by capillary electrophoresis. Journal of Agricultural and Food Chemistry. 47(11):4649-4652.

[8] Nakamura, Y., Ishimitsu, S. and Tonogai, Y. 2000.Effects of quercetin and rutin on serum and hepatic lipid concentrations, fecal steroid excretion and serum antioxidant properties. Journal of Health Science.46(4):229-240.

[9] Baccan, M. M., Chiarelli-Neto, O., Pereira, R. M. S. and Espósito, B. P. 2012.Quercetin as a shuttle for labile iron. Journal of Inorganic Biochemistry.107(1):34-39.

[10] Sethi, S., Haas, M., Markowitz, G. S., D’Agati, V. D., Rennke, H. G., Jennette, J. C. and Cosio, F. G. 2015. Mayo clinic/renal pathology society consensus report on pathologic classification, diagnosis, and reporting of GN. Journal of the American Society of Nephrology, ASN-2015060612.

[11] Prakash, A. and Adhikari, D. 2011. Application of Schiff bases and their metal complexes-A Review. Int. J. Chem. Tech. Res, 3(4):1891-1896.

[12] Fu, W., Chen, J., Cai, Y., Lei, Y., Chen, L., Pei, L. and Ruan, J. 2010. Antioxidant, free radical scavenging, anti-inflammatory and hepatoprotective potential of the extract from Parathelypterisnipponica (Franch. etSav.) Ching. Journal of Ethnopharmacology, 130(3):521-528.

[13] Barku, V. Y. A., Opoku-Boahen, Y., Owusu-Ansah, E. and
Mensah, E. F.2013. Antioxidant activity and the estimation of total phenolic and flavonoid contents of the root extract of Amaranthusspinosus. Asian Journal of Plant Science and Research, 3(1): 69-74.

[14] Proestos, C., Lytoudi, K., Mavromelanidou, O. K., Zoumpoulakis, P. and Sinanoglou, V. J. 2013.Antioxidant capacity of selected plant extracts and their essential oils. Antioxidants, 2(1): 11-22.

[15] De Souza, R. F. and De Giovani, W. F. 2004.Antioxidant properties of complexes of flavonoids with metal ions. Redox Report, 9(2): 97-104.

[16] Mel'nikova, N. B., Ioffe, I. D. and Tsareva, L. A. 2002. Reaction of bioflavonoids with copper (II) acetate in aqueous solution. Chemistry of Natural Compounds, 38(1): 33-39.

[17] Malešev, D. and Kuntić, V. 2007.Investigation of metal-flavonoid chelates and the determination of flavonoids via metal-flavonoid complexing reactions. Journal of the Serbian Chemical Society, 72(10):921-939.

[18] Yaul, S. R., Yaul, A. R., Pethe, G. B., and Aswar, A. S. 2009. Synthesis and characterization of transition metal complexes with N, O-chelating hydrazone Schiff base ligand. American-Eurasian Journal of Scientific Research, 4(4):229-234.

[19] Panhwar, Q. K. and Memon, S. 2014. Synthesis of Cr (III)-morin complex: characterization and antioxidant study. The Scientific World Journal.

[20] Roy, S., Mondal, P., Sengupta, P. S., Dhak, D., Santra, R. C., Das, S., and Guin, P. S. 2015.Spectroscopic, computational and electrochemical studies on the formation of the copper complex of 1-amino-4-hydroxy-9, 10-anthraquinone and effect of it on superoxide formation by NADH dehydrogenase. Dalton Transactions, 44(12): 5428-5440.

[21] Payán-Gómez, S. A., Flores-Holguín, N., Pérez-Hernández, A., Piñón-Miramontes, M., \& Glossman-Mitnik, D. (2010). Computational molecular characterization of the flavonoid rutin. Chemistry Central Journal, 4(1), 12. 